\title{
Scorza-Dragoni approach to Dirichlet problem in Banach spaces
}

\author{
Jan Andres ${ }^{1 *}$, Luisa Malaguti ${ }^{2}$ and Martina Pavlačková
}

\author{
"Correspondence: \\ jan.andres@upol.cz \\ ${ }^{1}$ Department of Mathematical \\ Analysis and Applications of \\ Mathematics, Faculty of Science, \\ Palacký University, 17. listopadu 12, \\ Olomouc, 771 46, Czech Republic \\ Full list of author information is \\ available at the end of the article
}

\begin{abstract}
Hartman-type conditions are presented for the solvability of a multivalued Dirichlet problem in a Banach space by means of topological degree arguments, bounding functions, and a Scorza-Dragoni approximation technique. The required transversality conditions are strictly localized on the boundaries of given bound sets. The main existence and localization result is applied to a partial integro-differential equation involving possible discontinuities in state variables. Two illustrative examples are supplied. The comparison with classical single-valued results in this field is also made. MSC: 34A60; 34B15; 47H04
\end{abstract}

Keywords: Dirichlet problem; Scorza-Dragoni-type technique; strictly localized bounding functions; solutions in a given set; condensing multivalued operators

\section{Introduction}

In this paper, we will establish sufficient conditions for the existence and localization of strong solutions to a multivalued Dirichlet problem in a Banach space via degree arguments combined with a bound sets technique. More precisely, Hartman-type conditions (cf. [1]), i.e. sign conditions w.r.t. the first state variable and growth conditions w.r.t. the second state variable, will be presented, provided the right-hand side is a multivalued upper-Carathéodory mapping which is $\gamma$-regular w.r.t. the Hausdorff measure of noncompactness $\gamma$.

The main aim will be two-fold: (i) strict localization of sign conditions on the boundaries of bound sets by means of a technique originated by Scorza-Dragoni [2], and (ii) the application of the obtained abstract result (see Theorem 3.1 below) to an integro-differential equation involving possible discontinuities in a state variable. The first aim allows us, under some additional restrictions, to extend our earlier results obtained for globally upper semicontinuous right-hand sides and partly improve those for upper-Carathéodory righthand sides (see [3]). As we shall see, the latter aim justifies such an abstract setting, because the problem can be transformed into the form of a differential inclusion in a Hilbert $L^{2}$ space. Roughly speaking, problems of this type naturally require such an abstract setting. In order to understand in a deeper way what we did and why, let us briefly recall classical results in this field and some of their extensions.

Hence, consider firstly the Dirichlet problem in the simplest vector form:

$$
\left.\begin{array}{l}
\ddot{x}(t)=f(t, x(t), \dot{x}(t)), \quad t \in[0,1], \\
x(1)=x(0)=0,
\end{array}\right\}
$$

O2014 Andres et al.; licensee Springer. This is an Open Access article distributed under the terms of the Creative Commons Attribution License (http://creativecommons.org/licenses/by/2.0), which permits unrestricted use, distribution, and reproduction in any medium, provided the original work is properly cited. 
where $f:[0,1] \times \mathbb{R}^{n} \times \mathbb{R}^{n} \rightarrow \mathbb{R}^{n}$ is, for the sake of simplicity allowing the comparison of the related results, a continuous function.

The first existence results, for a bounded $f$ in (1), are due to Scorza-Dragoni [4, 5]. Let us note that his name in the title is nevertheless related to the technique developed in [2] rather than to the existence results in $[4,5]$.

It is well known (see e.g. [3, 6-13]) that the problem (1) is solvable on various levels of generality provided:

(i sign $) \quad \exists R>0$ such that $\langle f(t, x, y), x\rangle>0$, for $(t, x, y) \in[0,1] \times \mathbb{R}^{n} \times \mathbb{R}^{n}$ with $\|x\|=R$,

(ii growth) $\exists C_{1} \geq 0, C_{2} \geq 0$ such that $C_{1} R<1$ and $\|f(t, x, y)\| \leq C_{1}\|y\|^{2}+C_{2}$, for $(t, x, y) \in$ $[0,1] \times \mathbb{R}^{n} \times \mathbb{R}^{n}$ with $\|x\| \leq R$.

Let us note that the existence of the same constant $R>0$ in $\left(\mathrm{i}_{\text {sign }}\right)$ and (ii $\left.\mathrm{i}_{\text {growth }}\right)$ can be assumed either explicitly as in $[6,7,9,11,13]$ or it follows from the assumptions as those in $[8,10,12]$.

(1) Hartmann [9] ( $c f$. also [1]) generalized both conditions as follows:

(i $\left.\mathrm{i}_{\mathrm{H}}\right) \exists R>0$ such that $\langle f(t, x, y), x\rangle+\|y\|^{2}>0$, for $t \in[0,1]$ and $(x, y) \in \mathbb{R}^{n} \times \mathbb{R}^{n}$ such that $\|x\|=R$ and $\langle x, y\rangle=0$,

$\left(\mathrm{i}_{\mathrm{H}}\right)$ the well-known Bernstein-Nagumo-Hartman condition (for its definition and more details, see e.g. $[1,14])$.

Let us note that the strict inequality in $\left(\mathrm{i}_{\mathrm{H}}\right)$ can be replaced by a non-strict one (see e.g. [1, Chapter XII,II,5], [11, Corollary 6.2]).

(2) Lasota and Yorke [10] improved condition ( $\mathrm{i}_{\text {sign }}$ ) with suitable constants $K_{1} \geq 0$ and $K_{2}>0$ in the following way:

(i $\left.\mathrm{i}_{\mathrm{LY}}\right)\langle f(t, x, y), x\rangle+\|y\|^{2} \geq-K_{1}(1+\|x\|+\langle x, y\rangle)+K_{2}\|y\|$,

but for $t \in[0,1],(x, y) \in \mathbb{R}^{n} \times \mathbb{R}^{n}$, and replaced (ii $\mathrm{i}_{\text {growth }}$ ) by the Bernstein-NagumoHartman condition.

Since $\left(\mathrm{i}_{\mathrm{LY}}\right)$ implies ( $\left.c f .[10]\right)$ the existence of a constant $K \geq 0$ such that

$$
\langle f(t, x, y), x\rangle+\|y\|^{2} \geq-K(1+\|x\|+|\langle x, y\rangle|),
$$

for $(t, x, y) \in[0,1] \times \mathbb{R}^{n} \times \mathbb{R}^{n}$, the sign condition ( $\left.\mathrm{i}_{\mathrm{LY}}\right)$ is obviously more liberal than ( $\left.\mathrm{i}_{\text {sign }}\right)$ as well as than $\left(\mathrm{i}_{\mathrm{H}}\right)$, on the intersection of their domains.

If $K_{1}>0$ in ( $\mathrm{i}_{\mathrm{LY}}$ ), then constant $K_{2}$ can be even equal to zero, i.e. $K_{2}=0$, in ( $\mathrm{i}_{\mathrm{LY}}$ ) (see e.g. [7, Corollary V.26 on p.74]). Moreover, the related Bernstein-Nagumo-Hartman condition can only hold for $x$ in a suitable convex, closed, bounded subset of $\mathbb{R}^{n}$ (see again e.g. [7]).

(3) Following the ideas of Mawhin in [7, 11, 12], Amster and Haddad [6] demonstrated that an open, bounded subset of $\mathbb{R}^{n}$, say $D \subset \mathbb{R}^{n}$, need not be convex, provided it has a $C^{2}$-boundary $\partial D$ such that condition $\left(\mathrm{i}_{\mathrm{H}}\right)$ can be generalized as follows:

$\left(\mathrm{i}_{\mathrm{AH}}\right)\left\langle f(t, x, y), n_{x}\right\rangle \geq I_{x}(y),(t, x, y) \in[0,1] \times T \partial D \times \mathbb{R}^{n}$, with $\left\langle n_{x}, y\right\rangle=0$

where $n_{x}$ is the outer-pointing normal unit vector field, $T \partial D$ denotes the tangent vector bundle and $I_{x}(y)$ stands for the second fundamental form of the hypersurface. 
Since for the ball $D:=B(0, R), R>0$, we can have

$$
I_{x}(y)=-\frac{\|y\|^{2}}{R} \quad \text { and } \quad n_{x}=\frac{x}{R}
$$

condition $\left(\mathrm{i}_{\mathrm{AH}}\right)$ is obviously more general than the original Hartman condition $\left(\mathrm{i}_{\mathrm{H}}\right)$.

Nevertheless, the growth condition takes there only the form (ii ${ }_{\text {growth }}$ ), namely with $\|x\| \leq R$ replaced by $x \in \bar{D}$, where $R$ denotes, this time, the radius of $D$.

For a convex, open, bounded subset $D \subset \mathbb{R}^{n}$, the particular case of $\left(\mathrm{i}_{\mathrm{AH}}\right)$ can read as follows:

(i conv $_{\text {) }}\left\langle f(t, x, y), n_{x}\right\rangle>0$, for $(t, x, y) \in[0,1] \times \mathbb{R}^{n} \times \mathbb{R}^{n}$ with $x \in \partial D$ and $\left\langle n_{x}, y\right\rangle=0$,

which is another well-known generalization of $\left(i_{\text {sign }}\right)$.

(4) In a Hilbert space $H$, for a completely continuous mapping $f$, Mawhin [12] has shown that, for real constants $a, b, c$ such that $a+b<1$, condition ( $\mathrm{i}_{\text {sign }}$ ) can be replaced in particular by

(i $\left.\mathrm{i}_{\mathrm{M}}\right)\langle f(t, x, y), x\rangle \geq-\left(a\|x\|^{2}+b\|x\|\|y\|+c\|x\|\right),(t, x, y) \in[0,1] \times H \times H$,

and (ii ${ }_{\text {growth}}$ ) by an appropriate version of the Bernstein-Nagumo-Hartman condition.

(5) In a Banach space $E$, Schmitt and Thompson [13] improved, for a completely continuous mapping $f$, condition ( $\left.\mathrm{i}_{\text {conv }}\right)$ in the sense that the strict inequality in ( $\left.\mathrm{i}_{\text {conv }}\right)$ can be replaced by a non-strict one. More concretely, if there exists a convex, open, bounded subset $D \subset E$ of $E$ with $0 \in D$ such that

(isT) $\left\langle f(t, x, y), n_{x}\right\rangle \geq 0$, for $(t, x, y) \in[0,1] \times E \times E$, with $x \in \partial D$ and $\left\langle n_{x}, y\right\rangle=0$,

where $\langle\cdot, \cdot\rangle$ denotes this time the pairing between $E$ and its dual $E^{\prime}$, jointly with the appropriate Bernstein-Nagumo-Hartman condition, then the problem (1) admits a solution whose values are located in $\bar{D}$ (see [13, Theorem 4.1]).

In the Carathéodory case of $f:[0,1] \times \mathbb{R}^{n} \times \mathbb{R}^{n} \rightarrow \mathbb{R}^{n}$ in (1), for instance, the strict inequality in condition $\left(i_{\text {sign }}\right)$ can be replaced, according to [8, Theorem 6.1], by a non-strict one and the constants $C_{1}, C_{2}$ can be replaced without the requirement $C_{1} R<1$, but globally in $[0,1] \times \mathbb{R}^{n} \times \mathbb{R}^{n}$, by functions $c_{1}(t, x), c_{2}(t, x)$ which are bounded on bounded sets. Moreover, system (1) can be additively perturbed, for the same goal, by another Carathéodory function which is sublinear in both states variables $x$ and $y$.

On the other hand, the Carathéodory case brings about some obstructions in a strict localization of sign conditions on the boundaries of bound sets (see e.g. $[3,15])$. The same is also true for other boundary value problems (for Floquet problems, see e.g. [16-18]). Therefore, there naturally exist some extensions of classical results in this way. Further extensions concern problems in abstract spaces, functional problems, multivalued problems, etc. For the panorama of results in abstract spaces, see e.g. [19], where multivalued problems are also considered.

Nevertheless, let us note that in abstract spaces, it is extremely difficult (if not impossible) to avoid the convexity of given bound sets, provided the degree arguments are applied for non-compact maps (for more details, see [20]).

In this light, we would like to modify in the present paper the Hartman-type conditions $\left(i_{\text {sign }}\right)$, ( $\left.i_{\text {growth }}\right)$ at least in the following way: 
- the given space $E$ to be Banach (or, more practically, Hilbert),

- the right-hand side to be a multivalued upper-Carathéodory mapping $F$ which is $\gamma$-regular w.r.t. $(x, y) \in E \times E$ and either globally measurable or globally quasi-compact,

- the inequality in $\left(\mathrm{i}_{\mathrm{sign}}\right)$ to hold w.r.t. $x$ strictly on the boundary $\partial D$ of a convex, bounded subset $D \subset E$ (or, more practically, of the ball $B(0, R) \subset E$ ),

- condition (ii growth) to be replaced by a suitable growth condition which would allow us reasonable applications (the usage of the Bernstein-Nagumo-Hartman-type condition will be employed in this context by ourselves elsewhere).

Hence, let $E$ be a separable Banach space (with the norm $\|\cdot\|$ ) satisfying the RadonNikodym property (e.g. reflexivity, see e.g. [21, pp.694-695]) and let us consider the Dirichlet boundary value problem (b.v.p.)

$$
\left.\begin{array}{l}
\ddot{x}(t) \in F(t, x(t), \dot{x}(t)), \quad \text { for a.a. } t \in[0, T], \\
x(T)=x(0)=0,
\end{array}\right\}
$$

where $F:[0, T] \times E \times E \multimap E$ is an upper-Carathéodory multivalued mapping.

Let us note that in the entire paper all derivatives will be always understood in the sense of Fréchet and, by the measurability, we mean the one with respect to the Lebesgue $\sigma$-algebra in $[0, T]$ and the Borel $\sigma$-algebra in $E$.

The notion of a solution will be understood in a strong (i.e. Carathéodory) sense. Namely, by a solution of problem (2) we mean a function $x:[0, T] \rightarrow E$ whose first derivative $\dot{x}(\cdot)$ is absolutely continuous and satisfies (2), for almost all $t \in[0, T]$.

The solution of the b.v.p. (2) will be obtained as the limit of a sequence of solutions of approximating problems that we construct by means of a Scorza-Dragoni-type result developed in [22]. The approximating problems will be treated by means of the continuation principle developed in [19].

\section{Preliminaries}

Let $E$ be as above and $[0, T] \subset \mathbb{R}$ be a closed interval. By the symbol $L^{1}([0, T], E)$, we shall mean the set of all Bochner integrable functions $x:[0, T] \rightarrow E$. For the definition and properties of Bochner integrals, see e.g. [21, pp.693-701]. The symbol $A C^{1}([0, T], E)$ will be reserved for the set of functions $x:[0, T] \rightarrow E$ whose first derivative $\dot{x}(\cdot)$ is absolutely continuous. Then $\ddot{x} \in L^{1}([0, T], E)$ and the fundamental theorem of calculus (the NewtonLeibniz formula) holds (see e.g. [21, pp.695-696], [23, pp.243-244]). In the sequel, we shall always consider $A C^{1}([0, T], E)$ as a subspace of the Banach space $C^{1}([0, T], E)$ and by the symbol $\mathcal{L}(E)$ we shall mean the Banach space of all linear, bounded transformations $L$ : $E \rightarrow E$ endowed with the sup-norm.

Given $C \subset E$ and $\varepsilon>0$, the symbol $B(C, \varepsilon)$ will denote, as usually, the set $C+\varepsilon B$, where $B$ is the open unit ball in $E$ centered at 0 , i.e. $B=\{x \in E \mid\|x\|<1\}$. In what follows, the symbol $\mu$ will denote the Lebesgue measure on $\mathbb{R}$.

Let $E^{\prime}$ be the Banach space dual to $E$ and let us denote by $\left.\langle\cdot, \cdot\rangle\right\rangle$ the pairing (the duality relation) between $E$ and $E^{\prime}$, i.e., for all $\Phi \in E^{\prime}$ and $x \in E$, we put $\Phi(x)=:\langle\Phi, x\rangle$.

We recall also the Pettis measurability theorem which will be used in Section 4 and which we state here in the form of proposition. 
Proposition 2.1 [24, p.278] Let $(X, \Sigma)$ be a measure space, E be a separable Banach space. Then $f: X \rightarrow E$ is measurable if and only if for every $e \in E^{\prime}$ the function $e \circ f: X \rightarrow \mathbb{R}$ is measurable with respect to $\Sigma$ and the Borel $\sigma$-algebra in $\mathbb{R}$.

We shall also need the following definitions and notions from multivalued analysis. Let $X, Y$ be two metric spaces. We say that $F$ is a multivalued mapping from $X$ to $Y$ (written $F: X \multimap Y)$ if, for every $x \in X$, a non-empty subset $F(x)$ of $Y$ is given. We associate with $F$ its graph $\Gamma_{F}$, the subset of $X \times Y$, defined by $\Gamma_{F}:=\{(x, y) \in X \times Y \mid y \in F(x)\}$.

A multivalued mapping $F: X \multimap Y$ is called upper semicontinuous (shortly, u.s.c.) if, for each open subset $U \subset Y$, the set $\{x \in X \mid F(x) \subset U\}$ is open in $X$.

Let $J \subset \mathbb{R}$ be a compact interval. A mapping $F: J \multimap Y$, where $Y$ is a separable metric space, is called measurable if, for each open subset $U \subset Y$, the set $\{t \in J \mid F(t) \subset U\}$ belongs to a $\sigma$-algebra of subsets of $J$.

A multivalued mapping $F: X \multimap Y$ is called compact if the set $F(X)=\bigcup_{x \in X} F(x)$ is contained in a compact subset of $Y$ and it is called quasi-compact if it maps compact sets onto relatively compact sets.

Let $J \subset \mathbb{R}$ be a given compact interval. A multivalued mapping $F: J \times X \multimap Y$, where $Y$ is a separable Banach space, is called an upper-Carathéodory mapping if the map $F(\cdot, x)$ : $J \multimap Y$ is measurable, for all $x \in X$, the map $F(t, \cdot): X \multimap Y$ is u.s.c., for almost all $t \in J$, and the set $F(t, x)$ is compact and convex, for all $(t, x) \in J \times X$.

The technique that will be used for proving the existence and localization result consists in constructing a sequence of approximating problems. This construction will be made on the basis of the Scorza-Dragoni-type result developed in [22] ( $c f$. also [25]).

For more details concerning multivalued analysis, see e.g. [23, 26, 27].

Definition 2.1 An upper-Carathéodory mapping $F:[0, T] \times X \times X \multimap X$ is said to have the Scorza-Dragoni property if there exists a multivalued mapping $F_{0}:[0, T] \times X \times X \multimap$ $X \cup\{\emptyset\}$ with compact, convex values having the following properties:

(i) $F_{0}(t, x, y) \subset F(t, x, y)$, for all $(t, x, y) \in[0, T] \times X \times X$,

(ii) if $u, v:[0, T] \rightarrow X$ are measurable functions with $v(t) \in F(t, u(t), \dot{u}(t))$, for a.a. $t \in[0, T]$, then also $v(t) \in F_{0}(t, u(t), \dot{u}(t))$, for a.a. $t \in[0, T]$,

(iii) for every $\varepsilon>0$, there exists a closed $I_{\varepsilon} \subset[0, T]$ such that $\mu\left([0, T] \backslash I_{\varepsilon}\right)<\varepsilon$, $F_{0}(t, x, y) \neq \emptyset$, for all $(t, x, y) \in I_{\varepsilon} \times X \times X$, and $F_{0}$ is u.s.c. on $I_{\varepsilon} \times X \times X$.

The following two propositions are crucial in our investigation. The first one is almost a direct consequence of the main result in [22] (cf. [25] and [16, Proposition 2]). The second one allows us to construct a sequence of approximating problems of (2).

Proposition 2.2 Let $E$ be a separable Banach space and $F:[0, T] \times E \times E \multimap E$ be an upper-Carathéodory mapping. If $F$ is globally measurable or quasi-compact, then $F$ has the Scorza-Dragoni property.

Proposition 2.3 ( $c f$. [18, Theorem 2.2]) Let E be a Banach space and $K \subset E$ a non-empty, open, convex, bounded set such that $0 \in K$. Moreover, let $\varepsilon>0$ and $V: E \rightarrow \mathbb{R}$ be a Fréchet differentiable function with $\dot{V}$ Lipschitzian in $\overline{B(\partial K, \varepsilon)}$ satisfying

(H1) $\left.V\right|_{\partial K}=0$,

(H2) $V(x) \leq 0$, for all $x \in \bar{K}$,

(H3) $\|\dot{V}(x)\| \geq \delta$, for all $x \in \partial K$, where $\delta>0$ is given. 
Then there exist $k \in(0, \varepsilon]$ and a bounded Lipschitzian function $\phi: \overline{B(\partial K, k)} \rightarrow$ E such that $\left\langle\dot{V}_{x}, \phi(x)\right\rangle=1$, for every $x \in \overline{B(\partial K, k)}$.

Remark 2.1 Let us note that the function $x \rightarrow \phi(x)\left\|\dot{V}_{x}\right\|$, where $\phi$ and $\dot{V}_{x}$ are the same as in Proposition 2.3, is Lipschitzian and bounded in $\overline{B(\partial K, k)}$. The symbol $\dot{V}_{x}$ denotes as usually the first Fréchet derivative of $V$ at $x$.

Example 2.1 If $V$ satisfies all the assumptions of Proposition 2.3, then it is easy to prove the existence of $\sigma \in(0, \varepsilon]$ such that $\left\|\dot{V}_{x}\right\| \geq \frac{\delta}{2}$, for all $x \in \overline{B(\partial K, \sigma)}$. Consequently, when $E$ is an arbitrary Hilbert space, we can define $\phi: \overline{B(\partial K, \sigma)} \rightarrow E$ by the formula

$$
\phi(x):=\frac{\nabla V(x)}{\|\nabla V(x)\|^{2}}
$$

which satisfies all the properties mentioned in Proposition 2.3.

Definition 2.2 Let $N$ be a partially ordered set, $E$ be a Banach space and let $P(E)$ denote the family of all non-empty bounded subsets of $E$. A function $\beta: P(E) \rightarrow N$ is called a measure of non-compactness (m.n.c.) in $E$ if $\beta(\overline{\operatorname{co} \Omega})=\beta(\Omega)$, for all $\Omega \in P(E)$, where $\overline{\operatorname{co} \Omega}$ denotes the closed convex hull of $\Omega$.

A m.n.c. $\beta$ is called:

(i) monotone if $\beta\left(\Omega_{1}\right) \leq \beta\left(\Omega_{2}\right)$, for all $\Omega_{1} \subset \Omega_{2} \subset E$,

(ii) non-singular if $\beta(\{x\} \cup \Omega)=\beta(\Omega)$, for all $x \in E$ and $\Omega \subset E$.

If $N$ is a cone in a Banach space, then a m.n.c. $\beta$ is called:

(iii) semi-homogeneous if $\beta(t \Omega)=|t| \beta(\Omega)$, for every $t \in \mathbb{R}$ and every $\Omega \subset E$,

(iv) regular when $\beta(\Omega)=0$ if and only if $\Omega$ is relatively compact,

(v) algebraically subadditive if $\gamma\left(\Omega_{1}+\Omega_{2}\right) \leq \gamma\left(\Omega_{1}\right)+\gamma\left(\Omega_{2}\right)$, for all $\Omega_{1}, \Omega_{2} \subset E$.

The typical example of an m.n.c. is the Hausdorff measure of non-compactness $\gamma$ defined, for all $\Omega \subset E$ by

$$
\gamma(\Omega):=\inf \left\{\varepsilon>0: \exists n \geq 1 \exists x_{1}, \ldots, x_{n} \in E: \Omega \subset \bigcup_{i=1}^{n} B\left(\left\{x_{i}\right\}, \varepsilon\right)\right\} .
$$

The Hausdorff m.n.c. is monotone, non-singular, semi-homogeneous and regular. Moreover, if $M \in \mathcal{L}(E)$ and $\Omega \subset E$, then (see, e.g., [27])

$$
\gamma(M \Omega) \leq\|M\|_{\mathcal{L}(E)} \gamma(\Omega) .
$$

Let $E$ be a separable Banach space and $\left\{f_{n}\right\}_{n} \subset L^{1}([0, T], E)$ be such that $\left\|f_{n}(t)\right\| \leq \alpha(t)$, $\gamma\left(\left\{f_{n}(t)\right\}_{n}\right) \leq c(t)$, for a.a. $t \in[0, T]$, all $n \in \mathbb{N}$ and suitable $\alpha, c \in L^{1}([0, T], \mathbb{R})$, then $(c f$. [27])

$$
\gamma\left(\left\{\int_{0}^{T} f_{n}(t) d t\right\}_{n}\right) \leq \int_{0}^{T} c(t) d t
$$

Moreover, if $h: E \multimap E$ is $L$-Lipschitzian, then

$$
\gamma(h(\Omega)) \leq L \gamma(\Omega)
$$

for all bounded $\Omega \subset E$. 
Furthermore, for all subsets $\Omega$ of $E$ (see e.g. [17]),

$$
\gamma\left(\bigcup_{\lambda \in[0,1]} \lambda \Omega\right)=\gamma(\Omega)
$$

Let us now introduce the function

$$
\begin{aligned}
\alpha(\Omega):= & \max _{\left\{w_{n}\right\}_{n} \subset \Omega}\left(\sup _{t \in[0, T]}\left[\gamma\left(\left\{w_{n}(t)\right\}_{n}\right)+\gamma\left(\left\{\dot{w}_{n}(t)\right\}_{n}\right)\right],\right. \\
& \left.\bmod _{C}\left(\left\{w_{n}\right\}_{n}\right)+\bmod _{C}\left(\left\{\dot{w}_{n}\right\}_{n}\right)\right),
\end{aligned}
$$

defined on the bounded $\Omega \subset C^{1}([0, T], E)$, where the ordering is induced by the positive cone in $\mathbb{R}^{2}$ and where $\bmod _{C}(\Omega)$ denotes the modulus of continuity of a subset $\Omega \subset C([0, T], E) .{ }^{\mathrm{a}}$ It was proved in [19] that the function $\alpha$ given by (7) is an m.n.c. in $C^{1}([0, T], E)$ that is monotone, non-singular and regular.

Definition 2.3 Let $E$ be a Banach space and $X \subset E$. A multivalued mapping $F: X \multimap E$ with compact values is called condensing with respect to an m.n.c. $\beta$ (shortly, $\beta$-condensing) if, for every bounded $\Omega \subset X$ such that $\beta(F(\Omega)) \geq \beta(\Omega)$, we see that $\Omega$ is relatively compact. A family of mappings $G: X \times[0,1] \multimap E$ with compact values is called $\beta$-condensing if, for every bounded $\Omega \subset X$ such that $\beta(G(\Omega \times[0,1])) \geq \beta(\Omega)$, we see that $\Omega$ is relatively compact.

The proof of the main result ( $c f$. Theorem 3.1 below) will be based on the following slight modification of the continuation principle developed in [19]. Since the proof of this modified version differs from the one in [19] only slightly in technical details, we omit it here.

Proposition 2.4 Let us consider the b.v.p.

$$
\left.\begin{array}{l}
\ddot{x}(t) \in \varphi(t, x(t), \dot{x}(t)), \quad \text { for a.a. } t \in[0, T], \\
x \in S,
\end{array}\right\}
$$

where $\varphi:[0, T] \times E \times E \multimap E$ is an upper-Carathéodory mapping and $S \subset A C^{1}([0, T], E)$. Let $H:[0, T] \times E \times E \times E \times E \times[0,1] \multimap E$ be an upper-Carathéodory mapping such that

$$
H(t, c, d, c, d, 1) \subset \varphi(t, c, d), \quad \text { for all }(t, c, d) \in[0, T] \times E \times E .
$$

Moreover, assume that the following conditions hold:

(i) There exist a closed set $S_{1} \subset S$ and a closed, convex set $Q \subset C^{1}([0, T], E)$ with a non-empty interior Int $Q$ such that each associated problem

$$
\left.P(q, \lambda) \begin{array}{l}
\ddot{x}(t) \in H(t, x(t), \dot{x}(t), q(t), \dot{q}(t), \lambda), \quad \text { for a.a. } t \in[0, T], \\
x \in S_{1},
\end{array}\right\}
$$

where $q \in Q$ and $\lambda \in[0,1]$, has a non-empty, convex set of solutions (denoted by $\mathfrak{T}(q, \lambda)$ ). 
(ii) For every non-empty, bounded set $\Omega \subset E \times E \times E \times E$, there exists $v_{\Omega} \in L^{1}([0, T],[0, \infty))$ such that

$$
\|H(t, x, y, u, v, \lambda)\| \leq v_{\Omega}(t)
$$

for a.a. $t \in[0, T]$ and all $(x, y, u, v) \in \Omega$ and $\lambda \in[0,1]$.

(iii) The solution mapping $\mathfrak{T}$ is quasi-compact and $\mu$-condensing with respect to a monotone and non-singular m.n.c. $\mu$ defined on $C^{1}([0, T], E)$.

(iv) For each $q \in Q$, the set of solutions of problem $P(q, 0)$ is a subset of $\operatorname{Int} Q$, i.e. $\mathfrak{T}(q, 0) \subset \operatorname{Int} Q$, for all $q \in Q$.

(v) For each $\lambda \in(0,1)$, the solution mapping $\mathfrak{T}(\cdot, \lambda)$ has no fixed points on the boundary $\partial Q$ of $Q$.

Then the b.v.p. (8) has a solution in $Q$.

\section{Main result}

Combining the foregoing continuation principle with the Scorza-Dragoni-type technique (cf. Proposition 2.2), we are ready to state the main result of the paper concerning the solvability and localization of a solution of the multivalued Dirichlet problem (2).

Theorem 3.1 Consider the Dirichlet b.v.p. (2). Suppose that $F:[0, T] \times E \times E \multimap E$ is an upper-Carathéodory mapping which is either globally measurable or quasi-compact. Furthermore, let $K \subset E$ be a non-empty, open, convex, bounded subset containing 0 of a separable Banach space E satisfying the Radon-Nikodym property. Let the following conditions $\left(2_{\mathrm{i}}\right)-\left(2_{\mathrm{iii}}\right)$ be satisfied:

$\left(2_{\mathrm{i}}\right) \quad \gamma\left(F\left(t, \Omega_{1} \times \Omega_{2}\right)\right) \leq g(t)\left(\gamma\left(\Omega_{1}\right)+\gamma\left(\Omega_{2}\right)\right)$, for a.a. $t \in[0, T]$ and each $\Omega_{1} \subset \bar{K}$, and each bounded $\Omega_{2} \subset E$, where $g \in L^{1}([0, T],[0, \infty))$ and $\gamma$ is the Hausdorff m.n.c. in $E$.

$\left(2_{\mathrm{ii}}\right)$ For every non-empty, bounded $\Omega \subset E$, there exists $v_{\Omega} \in L^{1}([0, T],[0, \infty))$ such that

$$
\|F(t, x, y)\| \leq v_{\Omega}(t)
$$

for a.a. $t \in[0, T]$ and all $(x, y) \in \Omega \times E$.

$\left(2_{\mathrm{iii}}\right)$

$$
(T+4)\|g\|_{L^{1}([0, T], \mathbb{R})}<4
$$

Furthermore, let there exist $\varepsilon>0$ and a function $V \in C^{2}(E, \mathbb{R})$, i.e. a twice continuously differentiable function in the sense of Fréchet, satisfying $(\mathrm{H} 1)-(\mathrm{H} 3)$ (cf. Proposition 2.3) with Fréchet derivative $\dot{V}$ Lipschitzian in $\overline{B(\partial K, \varepsilon)} .^{\mathrm{b}}$ Let there still exist $h>0$ such that

$$
\left\langle\ddot{V}_{x}(v), v\right\rangle \geq 0, \quad \text { for all } x \in B(\partial K, h), v \in E,
$$

where $\ddot{V}_{x}(v)$ denotes the second Fréchet derivative of $V$ at $x$ in the direction $(v, v) \in E \times E$. Finally, let

$$
\left\langle\dot{V}_{x}, w\right\rangle>0
$$

for a.a. $t \in(0, T)$ and all $x \in \partial K, v \in E$, and $w \in F(t, x, v)$. 
Then the Dirichlet b.v.p. (2) admits a solution whose values are located in $\bar{K}$.If, moreover, $0 \notin F(t, 0,0)$, for a.a. $t \in[0, T]$, then the obtained solution is non-trivial.

Proof Since the proof of this result is rather technical, it will be divided into several steps. At first, let us define the sequence of approximating problems. For this purpose, let $k$ be as in Proposition 2.3 and consider a continuous function $\tau: E \rightarrow[0,1]$ such that $\tau(x)=0$, for all $x \in E \backslash B(\partial K, k)$, and $\tau(x)=1$, for all $x \in \overline{B\left(\partial K, \frac{k}{2}\right)}$. According to Proposition 2.3 (see also Remark 2.1), the function $\hat{\phi}: E \rightarrow E$, where

$$
\hat{\phi}(x)= \begin{cases}\tau(x) \cdot \phi(x) \cdot\left\|\dot{V}_{x}\right\|, & \text { for all } x \in \overline{B(\partial K, k)}, \\ 0, & \text { for all } x \in E \backslash \overline{B(\partial K, k)},\end{cases}
$$

is well defined, continuous and bounded.

Since the mapping $(t, x, y) \multimap F(t, x, y)$ has, according to Proposition 2.2, the ScorzaDragoni property, we are able to find a decreasing sequence $\left\{J_{m}\right\}_{m}$ of subsets of $[0, T]$ and a mapping $F_{0}:[0, T] \times E \times E \multimap E \cup\{\emptyset\}$ with compact, convex values such that, for all $m \in \mathbb{N}$,

- $\mu\left(J_{m}\right)<\frac{1}{m}$

- $[0, T] \backslash J_{m}$ is closed,

- $(t, x, y) \multimap F_{0}(t, x, y)$ is u.s.c. on $[0, T] \backslash J_{m} \times E \times E$,

- $v_{\bar{K}}$ is continuous in $[0, T] \backslash J_{m}$ (cf.e.g. [2]).

If we put $J=\bigcap_{m=1}^{\infty} J_{m}$, then $\mu(J)=0, F_{0}(t, x, y) \neq \emptyset$, for all $t \in[0, T] \backslash J$, the mapping $(t, x, y) \multimap F_{0}(t, x, y)$ is u.s.c. on $[0, T] \backslash J \times E \times E$ and $v_{\bar{K}}$ is continuous in $[0, T] \backslash J$.

For each $m \in \mathbb{N}$, let us define the mapping $F_{m}:[0, T] \times E \times E \multimap E$ with compact, convex values by the formula

$$
F_{m}(t, x, y):= \begin{cases}F_{0}(t, x, y)+v_{\bar{K}}(t)\left(\chi_{J_{m}}(t)+\frac{1}{m}\right) \hat{\phi}(x), & \text { for all }(t, x, y) \in[0, T] \backslash J \times E \times E, \\ v_{\bar{K}}(t)\left(\chi_{J_{m}}(t)+\frac{1}{m}\right) \hat{\phi}(x), & \text { for all }(t, x, y) \in J \times E \times E .\end{cases}
$$

Let us consider the b.v.p.

$$
\begin{aligned}
& \left(P_{m}\right) \quad \begin{array}{l}
\ddot{x}(t) \in F_{m}(t, x(t), \dot{x}(t)), \quad \text { for a.a. } t \in[0, T] \\
x(T)=x(0)=0 .
\end{array}
\end{aligned}
$$

Now, let us verify the solvability of problems $\left(P_{m}\right)$. Let $m \in \mathbb{N}$ be fixed. Since $F_{0}$ is globally u.s.c. on $[0, T] \backslash J \times E \times E, F_{m}(\cdot, x, y)$ is measurable, for each $(x, y) \in E \times E$, and, due to the continuity of $\hat{\phi}, F_{m}(t, \cdot, \cdot)$ is u.s.c., for all $t \in[0, T] \backslash J$. Therefore, $F_{m}$ is an upper-Carathéodory mapping. Moreover, let us define the upper-Carathéodory mapping $H_{m}:[0, T] \times E \times E \times E \times E \times[0,1] \multimap E$ by the formula

$$
\begin{aligned}
& H_{m}(t, x, y, u, v, \lambda) \\
& \equiv H_{m}(t, u, v, \lambda) \\
& :=\left\{\begin{array}{lr}
\lambda F_{0}(t, u, v)+v_{\bar{K}}(t)\left(\chi_{J_{m}}(t)+\frac{1}{m}\right) \hat{\phi}(u), & \text { for all }(t, x, y, u, v, \lambda) \in[0, T] \backslash J \\
v_{\bar{K}}(t)\left(\chi_{J_{m}}(t)+\frac{1}{m}\right) \hat{\phi}(u), & \times E^{4} \times[0,1],
\end{array}\right. \\
&
\end{aligned}
$$


Let us show that, when $m \in \mathbb{N}$ is sufficiently large, all assumptions of Proposition 2.4 $\left(\right.$ for $\left.\varphi(t, x, \dot{x}):=F_{m}(t, x, \dot{x})\right)$ are satisfied.

For this purpose, let us define the closed set $S=S_{1}$ by

$$
S:=\left\{x \in A C^{1}([0, T], E): x(T)=x(0)=0\right\}
$$

and let the set $Q$ of candidate solutions be defined as $Q:=C^{1}([0, T], \bar{K})$. Because of the convexity of $K$, the set $Q$ is closed and convex.

For all $q \in Q$ and $\lambda \in[0,1]$, consider still the associated fully linearized problem

$$
\left.P_{m}(q, \lambda) \begin{array}{l}
\ddot{x}(t) \in H_{m}(t, q(t), \dot{q}(t), \lambda), \quad \text { for a.a. } t \in[0, T], \\
x(T)=x(0)=0,
\end{array}\right\}
$$

and denote by $\mathfrak{T}_{m}$ the solution mapping which assigns to each $(q, \lambda) \in Q \times[0,1]$ the set of solutions of $P_{m}(q, \lambda)$.

ad (i) In order to verify condition (i) in Proposition 2.4, we need to show that, for each $(q, \lambda) \in Q \times[0,1]$, the problem $P_{m}(q, \lambda)$ is solvable with a convex set of solutions. So, let $(q, \lambda) \in Q \times[0,1]$ be arbitrary and let $f_{q, \lambda}(\cdot)$ be a measurable selection of $H_{m}(\cdot, q(\cdot), \dot{q}(\cdot), \lambda)$, which surely exists (see, e.g., [27, Theorem 1.3.5]). According to $\left(2_{\mathrm{ii}}\right)$ and the definition of $H_{m}$, it is also easy to see that $f_{q, \lambda} \in L^{1}([0, T], E)$. The homogeneous problem corresponding to b.v.p. $P_{m}(q, \lambda)$,

$$
\left.\begin{array}{l}
\ddot{x}(t)=0, \quad \text { for a.a. } t \in[0, T], \\
x(T)=x(0)=0,
\end{array}\right\}
$$

has only the trivial solution, and therefore the single-valued Dirichlet problem

$$
\left.\begin{array}{l}
\ddot{x}(t)=f_{q, \lambda}(t), \quad \text { for a.a. } t \in[0, T], \\
x(T)=x(0)=0
\end{array}\right\}
$$

admits a unique solution $x_{q, \lambda}(\cdot)$ which is one of solutions of $P_{m}(q, \lambda)$. This is given, for a.a. $t \in[0, T]$, by $x_{q, \lambda}(t)=\int_{0}^{T} G(t, s) f_{q, \lambda}(s) d s$, where $G$ is the Green function associated to the homogeneous problem (12). The Green function $G$ and its partial derivative $\frac{\partial}{\partial t} G$ are defined by (cf. e.g. [28, pp.170-171])

$$
\begin{gathered}
G(t, s)= \begin{cases}\frac{(s-T) t}{T}, & \text { for all } 0 \leq t \leq s \leq T, \\
\frac{(t-T) s}{T}, & \text { for all } 0 \leq s \leq t \leq T,\end{cases} \\
\frac{\partial}{\partial t} G(t, s)= \begin{cases}\frac{(s-T)}{T}, & \text { for all } 0 \leq t<s \leq T, \\
\frac{s}{T}, & \text { for all } 0 \leq s<t \leq T .\end{cases}
\end{gathered}
$$

Thus, the set of solutions of $P_{m}(q, \lambda)$ is non-empty. The convexity of the solution sets follows immediately from the definition of $H_{m}$ and the fact that problems $P_{m}(q, \lambda)$ are fully linearized.

ad (ii) Let $\Omega \subset E \times E \times E \times E$ be bounded. Then, there exists a bounded $\Omega_{1} \subset E$ such that $\Omega \subset \Omega_{1} \times \Omega_{1} \times \Omega_{1} \times \Omega_{1}$ and, according to $\left(2_{\text {ii }}\right)$ and the definition of $H_{m}$, there exists 
$\hat{J} \subset[0, T]$ with $\mu(\hat{J})=0$ such that, for all $t \in[0, T] \backslash(J \cup \hat{J}),(x, y, u, v) \in \Omega$ and $\lambda \in[0,1]$,

$$
\left\|H_{m}(t, u, v, \lambda)\right\| \leq v_{\Omega_{1}}(t)+2 v_{\bar{K}}(t) \cdot \max _{x \in \bar{B}(\partial K, k)}\|\hat{\phi}(x)\| .
$$

Therefore, the mapping $H_{m}(t, q(t), \dot{q}(t), \lambda)$ satisfies condition (ii) from Proposition 2.4.

ad (iii) Since the verification of condition (iii) in Proposition 2.4 is technically the most complicated, it will be split into two parts: (iii ${ }_{1}$ ) the quasi-compactness of the solution operator $\mathfrak{T}_{m}$, (iii $\left.{ }_{2}\right)$ the condensity of $\mathfrak{T}_{m}$ w.r.t. the monotone and non-singular m.n.c. $\alpha$ defined by (7).

ad (iii $1_{1}$ ) Let us firstly prove that the solution mapping $\mathfrak{T}_{m}$ is quasi-compact. Since $C^{1}([0, T], E)$ is a complete metric space, it is sufficient to prove the sequential quasicompactness of $\mathfrak{T}_{m}$. Hence, let us consider the sequences $\left\{q_{n}\right\},\left\{\lambda_{n}\right\}, q_{n} \in Q, \lambda_{n} \in[0,1]$, for all $n \in \mathbb{N}$, such that $q_{n} \rightarrow q$ in $C^{1}([0, T], E)$ and $\lambda_{n} \rightarrow \lambda$. Moreover, let $x_{n} \in \mathfrak{T}_{m}\left(q_{n}, \lambda_{n}\right)$, for all $n \in \mathbb{N}$. Then there exists, for all $n \in \mathbb{N}, k_{n}(\cdot) \in F_{0}\left(\cdot, q_{n}(\cdot), \dot{q}_{n}(\cdot)\right)$ such that

$$
\ddot{x}_{n}(t)=f_{n}(t), \quad \text { for a.a. } t \in[0, T],
$$

where

$$
f_{n}(t)=\lambda_{n} k_{n}(t)+v_{\bar{K}}(t)\left(\chi_{J_{m}}(t)+\frac{1}{m}\right) \hat{\phi}\left(q_{n}(t)\right)
$$

and that

$$
x_{n}(T)=x_{n}(0)=0 .
$$

Since $q_{n} \rightarrow q$ and $\dot{q}_{n} \rightarrow \dot{q}$ in $C([0, T], E)$, there exists a bounded $\Omega \times \Omega \subset E \times E$ such that $\left(q_{n}(t), \dot{q}_{n}(t)\right) \in \Omega \times \Omega$, for all $t \in[0, T]$ and $n \in \mathbb{N}$. Therefore, there exists, according to condition $\left(2_{\mathrm{ii}}\right), v_{\Omega} \in L^{1}([0, T],[0, \infty))$ such that $\left\|f_{n}(t)\right\| \leq \varpi(t)$, for every $n \in \mathbb{N}$ and a.a. $t \in[0, T]$, where $\varpi(t):=v_{\Omega}(t)+2 v_{\bar{K}}(t) \cdot \max _{x \in \overline{B(\partial K, \varepsilon)}}\|\hat{\phi}(x)\|$.

Moreover, for every $n \in \mathbb{N}$ and a.a. $t \in[0, T]$,

$$
x_{n}(t)=\int_{0}^{T} G(t, s) f_{n}(s) d s
$$

and

$$
\dot{x}_{n}(t)=\int_{0}^{T} \frac{\partial}{\partial t} G(t, s) f_{n}(s) d s .
$$

Thus, $x_{n}$ satisfies, for every $n \in \mathbb{N}$ and a.a. $t \in[0, T],\left\|x_{n}(t)\right\| \leq a$ and $\left\|\dot{x}_{n}(t)\right\| \leq b$, where

$$
a:=\frac{T}{4} \int_{0}^{T} \varpi(s) d s \quad \text { and } \quad b:=\int_{0}^{T} \varpi(s) d s .
$$

Furthermore, for every $n \in \mathbb{N}$ and a.a. $t \in[0, T]$, we have

$$
\left\|\ddot{x}_{n}(t)\right\| \leq \varpi(t) .
$$

Hence, the sequences $\left\{x_{n}\right\}$ and $\left\{\dot{x}_{n}\right\}$ are bounded and $\left\{\ddot{x}_{n}\right\}$ is uniformly integrable. 
For each $t \in[0, T]$, the properties of the Hausdorff m.n.c. yield

$$
\begin{aligned}
\gamma\left(\left\{f_{n}(t)\right\}_{n}\right) \leq & \gamma\left(\left\{\lambda_{n} k_{n}(t)\right\}_{n}\right)+v_{\bar{K}}(t)\left(\chi_{I_{m}}(t)+\frac{1}{m}\right) \gamma\left(\left\{\hat{\phi}\left(q_{n}(t)\right)\right\}_{n}\right) \\
\leq & \gamma\left(\left\{k_{n}(t)\right\}_{n}\right)+v_{\bar{K}}(t)\left(\chi_{I_{m}}(t)+\frac{1}{m}\right) \\
& \times \gamma\left(\left\{\phi\left(q_{n}(t)\right)\left\|\dot{V}_{q_{n}(t)}\right\|: q_{n}(t) \in \overline{B(\partial K, \varepsilon)}\right\}\right) .
\end{aligned}
$$

Since $q_{n}(t) \in \bar{K}$, for all $t \in[0, T]$ and all $n \in \mathbb{N}$, it follows from condition $\left(2_{\mathrm{i}}\right)$ that, for a.a. $t \in[0, T]$

$$
\begin{aligned}
\gamma\left(\left\{f_{n}(t)\right\}_{n}\right) \leq & g(t)\left(\gamma\left(\left\{q_{n}(t)\right\}_{n}\right)+\gamma\left(\left\{\dot{q}_{n}(t)\right\}_{n}\right)\right) \\
& +v_{\bar{K}}(t)\left(\chi_{I_{m}}(t)+\frac{1}{m}\right) \gamma\left(\left\{\phi\left(q_{n}(t)\right)\left\|\dot{V}_{q_{n}(t)}\right\|: q_{n}(t) \in \overline{B(\partial K, \varepsilon)}\right\}\right) \\
\leq & g(t) \sup _{t \in[0, T]}\left(\gamma\left(\left\{q_{n}(t)\right\}_{n}\right)+\gamma\left(\left\{\dot{q}_{n}(t)\right\}_{n}\right)\right) \\
& +v_{\bar{K}}(t)\left(\chi_{I_{m}}(t)+\frac{1}{m}\right) \gamma\left(\left\{\phi\left(q_{n}(t)\right)\left\|\dot{V}_{q_{n}(t)}\right\|: q_{n}(t) \in \overline{B(\partial K, \varepsilon)}\right\}\right) .
\end{aligned}
$$

Since the function $x \rightarrow \phi(x)\left\|\dot{V}_{x}\right\|$ is Lipschitzian on $\overline{B(\partial K, \varepsilon)}$ with some Lipschitz constant $\hat{L}>0$ (see Remark 2.1), we get

$$
\gamma\left(\left\{f_{n}(t)\right\}_{n}\right) \leq\left(g(t)+\hat{L} v_{\bar{K}}(t)\left(\chi_{J_{m}}(t)+\frac{1}{m}\right)\right) \sup _{t \in[0, T]}\left(\gamma\left(\left\{q_{n}(t)\right\}_{n}\right)+\gamma\left(\left\{\dot{q}_{n}(t)\right\}_{n}\right)\right) .
$$

Since $q_{n} \rightarrow q$ and $\dot{q}_{n} \rightarrow \dot{q}$ in $C([0, T], E)$, we get, for all $t \in[0, T], \gamma\left(\left\{q_{n}(t)\right\}_{n}\right)=\gamma\left(\left\{\dot{q}_{n}(t)\right\}_{n}\right)=$ 0 , which implies that $\gamma\left(\left\{f_{n}(t)\right\}_{n}\right)=0$, for all $t \in[0, T]$.

For all $(t, s) \in[0, T] \times[0, T]$, the sequence $\left\{G(t, s) f_{n}(s)\right\}$ is relatively compact as well since, according to the semi-homogeneity of the Hausdorff m.n.c.,

$$
\gamma\left(\left\{G(t, s) f_{n}(s)\right\}\right) \leq|G(t, s)| \gamma\left(\left\{f_{n}(s)\right\}\right)=0, \quad \text { for all }(t, s) \in[0, T] \times[0, T] .
$$

Moreover, by means of (4) and (18),

$$
\gamma\left(\left\{x_{n}(t)\right\}\right)=\gamma\left(\left\{\int_{0}^{T} G(t, s) f_{n}(s) d s\right\}\right)=0, \quad \text { for all } t \in[0, T] .
$$

By similar reasoning, we also get

$$
\gamma\left(\left\{\dot{x}_{n}(t)\right\}\right)=0, \quad \text { for all } t \in[0, T]
$$

by which $\left\{x_{n}(t)\right\},\left\{\dot{x}_{n}(t)\right\}$ are relatively compact, for all $t \in[0, T]$.

Moreover, since $x_{n}$ satisfies for all $n \in \mathbb{N}(13),\left\{\ddot{x}_{n}(t)\right\}$ is relatively compact, for a.a. $t \in$ $[0, T]$. Thus, according to [23, Lemma III.1.30], there exist a subsequence of $\left\{\dot{x}_{n}\right\}$, for the sake of simplicity denoted in the same way as the sequence, and $x \in C^{1}([0, T], E)$ such that $\left\{\dot{x}_{n}\right\}$ converges to $\dot{x}$ in $C([0, T], E)$ and $\left\{\ddot{x}_{n}\right\}$ converges weakly to $\ddot{x}$ in $L^{1}([0, T], E)$. According 
to the classical closure results (cf. e.g. [27, Lemma 5.1.1]), $x \in \mathfrak{T}_{m}(q, \lambda)$, which implies the quasi-compactness of $\mathfrak{T}_{m}$.

ad (iii ${ }_{2}$ ) In order to show that, for $m \in \mathbb{N}$ sufficiently large, $\mathfrak{T}_{m}$ is $\alpha$-condensing with respect to the m.n.c. $\alpha$ defined by (7), let us consider a bounded subset $\Theta \subset Q$ such that $\alpha\left(\mathfrak{T}_{m}(\Theta \times[0,1])\right) \geq \alpha(\Theta)$. Let $\left\{x_{n}\right\} \subset \mathfrak{T}_{m}(\Theta \times[0,1])$ be a sequence such that

$$
\begin{aligned}
\alpha & \left(\mathfrak{T}_{m}(\Theta \times[0,1])\right) \\
& =\left(\sup _{t \in[0, T]}\left[\gamma\left(\left\{x_{n}(t)\right\}_{n}\right)+\gamma\left(\left\{\dot{x}_{n}(t)\right\}_{n}\right)\right], \bmod _{C}\left(\left\{x_{n}\right\}_{n}\right)+\bmod _{C}\left(\left\{\dot{x}_{n}\right\}_{n}\right)\right) .
\end{aligned}
$$

At first, let us show that the set $\mathfrak{T}_{m}(\Theta \times[0,1])$ is bounded. If $x \in \mathfrak{T}_{m}(\Theta \times[0,1])$, then there exist $q \in \Theta, \lambda \in[0,1]$ and $k(\cdot) \in F_{0}(\cdot, q(\cdot), \dot{q}(\cdot))$ such that

$$
x(t)=\int_{0}^{T} G(t, s) f(s) d s, \quad \dot{x}(t)=\int_{0}^{T} \frac{\partial G(t, s)}{\partial t} f(s) d s, \quad \text { for all } t \in[0, T],
$$

with $f(t)=\lambda k(t)+v_{\bar{K}}(t)\left(\chi_{I_{m}}(t)+\frac{1}{m}\right) \hat{\phi}(q(t))$, for a.a. $t \in[0, T]$.

Since $\Theta$ is bounded, there exists $\Omega \subset E$ such that $q(t) \in \Omega$, for all $q \in \Theta$ and all $t \in[0, T]$. Hence, according to $\left(2_{\mathrm{ii}}\right)$, there exists $v_{\Omega} \in L^{1}([0, T])$ such that $\|k(t)\| \leq v_{\Omega}(t)$, for a.a. $t \in$ $[0, T]$. Consequently

$$
\begin{aligned}
\|x(t)\|_{E} & \leq \max _{(t, s) \in[0,1] \times[0,1]}|G(t, s)|\left[\int_{0}^{T} v_{\Omega}(s) d s+2 \max _{x \in \bar{B}(\partial K, k)}\|\hat{\phi}(x)\| \int_{0}^{T} v_{\bar{K}}(t)\right] \\
& \leq \frac{T}{4}\left\|v_{\Omega}\right\|+2 \max _{x \in \bar{B}(\partial K, k)}\|\hat{\phi}(x)\| \cdot\left\|v_{\bar{K}}\right\| .
\end{aligned}
$$

Similarly,

$$
\begin{aligned}
\|\dot{x}(t)\|_{E} & \leq \max _{(t, s) \in[0,1] \times[0,1]}\left|\frac{\partial G(t, s)}{\partial}\right|\left[\int_{0}^{T}\|k(s)\| d s+2 \max _{x \in \overline{B(\partial K, k)}}\|\hat{\phi}(x)\| \int_{0}^{T} v_{\bar{K}}(t)\right] \\
& \leq\left\|v_{\Omega}\right\|+2 \max _{x \in \overline{B(\partial K, k)}}\|\hat{\phi}(x)\| \cdot\left\|\nu_{\bar{K}}\right\| .
\end{aligned}
$$

Thus, the set $\mathfrak{T}_{m}(\Theta \times[0,1])$ is bounded.

Moreover, we can find $\left\{q_{n}\right\} \subset \Theta,\left\{\lambda_{n}\right\} \subset[0,1]$ and $\left\{k_{n}\right\}$ satisfying, for a.a. $t \in[0, T]$, $k_{n}(t) \in F_{0}\left(t, q_{n}(t), \dot{q}_{n}(t)\right)$, such that, for all $t \in[0, T], x_{n}(t)$ and $\dot{x}_{n}(t)$ are defined by (15) and (16), respectively, where $f_{n}(t)$ is defined by (14).

By similar reasoning as in the part $a d\left(\mathrm{iii}_{1}\right)$, we obtain

$$
\gamma\left(\left\{f_{n}(t)\right\}_{n}\right) \leq\left(g(t)+\hat{L} v_{\bar{K}}(t)\left(\chi_{J_{m}}(t)+\frac{1}{m}\right)\right) \sup _{t \in[0, T]}\left(\gamma\left(\left\{q_{n}(t)\right\}_{n}\right)+\gamma\left(\left\{\dot{q}_{n}(t)\right\}_{n}\right)\right),
$$

for a.a. $t \in[0, T]$, and that

$$
\left\|f_{n}(t)\right\| \leq\left\|k_{n}(t)\right\|+2 \cdot \max _{x \in \bar{B}(\partial K, \varepsilon)}\|\hat{\phi}(x)\| \cdot v_{\bar{K}}(t), \quad \text { for a.a. } t \in[0, T] \text { and all } n \in \mathbb{N} .
$$

Since $k_{n}(t) \in F_{0}\left(t, q_{n}(t), \dot{q}_{n}(t)\right)$, for a.a. $t \in[0, T]$, and $q_{n} \in \Theta$, for all $n \in \mathbb{N}$, where $\Theta$ is a bounded subset of $C^{1}([0, T], E)$, there exists $\Omega \subset \bar{K}$ such that $q_{n}(t) \in \Omega$, for all $n \in \mathbb{N}$ and 
$t \in[0, T]$. Hence, it follows from condition $\left(2_{\mathrm{ii}}\right)$ that

$$
\left\|f_{n}(t)\right\| \leq v_{\Omega}(t)+2 \cdot v_{\bar{K}}(t) \cdot \max _{x \in \overline{B(\partial K, \varepsilon)}}\|\hat{\phi}(x)\|, \quad \text { for a.a. } t \in[0, T] .
$$

This implies $\left\|G(t, s) f_{n}(t)\right\| \leq|G(t, s)|\left(\nu_{\Omega}(t)+2 \cdot v_{\bar{K}}(t) \cdot \max _{x \in \overline{B(\partial K, \varepsilon)}}\|\hat{\phi}(x)\|\right)$, for a.a. $t, s \in$ $[0, T]$ and all $n \in \mathbb{N}$.

Moreover, by virtue of the semi-homogeneity of the Hausdorff m.n.c., for all $(t, s) \in$ $[0, T] \times[0, T]$, we have

$$
\begin{aligned}
\gamma\left(\left\{G(t, s) f_{n}(s)\right\}_{n}\right) \leq & |G(t, s)| \gamma\left(\left\{f_{n}(s)\right\}_{n}\right) \leq \frac{T}{4} \gamma\left(\left\{f_{n}(s)\right\}_{n}\right) \\
\leq & \frac{T}{4}\left(g(t)+\hat{L} v_{\bar{K}}(t)\left(\chi_{J_{m}}(t)+\frac{1}{m}\right)\right) \\
& \times \sup _{t \in[0, T]}\left(\gamma\left(\left\{q_{n}(t)\right\}_{n}\right)+\gamma\left(\left\{\dot{q}_{n}(t)\right\}_{n}\right)\right) .
\end{aligned}
$$

Let us denote

$$
\mathcal{S}:=\sup _{t \in[0, T]}\left(\gamma\left(\left\{q_{n}(t)\right\}_{n}\right)+\gamma\left(\left\{\dot{q}_{n}(t)\right\}_{n}\right)\right)
$$

and

$$
\mathcal{S}^{*}:=\sup _{t \in[0, T]}\left(\gamma\left(\left\{x_{n}(t)\right\}_{n}\right)+\gamma\left(\left\{\dot{x}_{n}(t)\right\}_{n}\right)\right) .
$$

According to (4) and (15) we thus obtain for each $t \in[0, T]$,

$$
\begin{aligned}
\gamma\left(\left\{x_{n}(t)\right\}_{n}\right) & =\gamma\left(\left\{\int_{0}^{T} G(t, s) f_{n}(s) d s\right\}_{n}\right) \\
& \leq \frac{T}{4}\left(\|g\|_{L^{1}}+\hat{L}\left(\left\|v_{\bar{K}}\right\|_{L^{1}\left(J_{m}\right)}+\frac{1}{m}\left\|v_{\bar{K}}\right\|_{L^{1}}\right)\right) \mathcal{S} .
\end{aligned}
$$

By similar reasonings, we can see that, for each $t \in[0, T]$,

$$
\gamma\left(\left\{\dot{x}_{n}(t)\right\}_{n}\right) \leq\left(\|g\|_{L^{1}}+\hat{L}\left(\left\|v_{\bar{K}}\right\|_{L^{1}\left(J_{m}\right)}+\frac{1}{m}\left\|v_{\bar{K}}\right\|_{L^{1}}\right)\right) \mathcal{S},
$$

when starting from condition (16). Subsequently,

$$
\mathcal{S}^{*} \leq \frac{T+4}{4}\left(\|g\|_{L^{1}}+\hat{L}\left(\left\|v_{\bar{K}}\right\|_{L^{1}\left(J_{m}\right)}+\frac{1}{m}\left\|v_{\bar{K}}\right\|_{L^{1}}\right)\right) \mathcal{S} .
$$

Since we assume that $\alpha\left(\mathfrak{T}_{m}(\Theta \times[0,1])\right) \geq \alpha(\Theta)$ and $\left\{q_{n}\right\}_{n} \subset \Theta$, we get

$$
\mathcal{S} \leq \mathcal{S}^{*} \leq \frac{T+4}{4}\left(\|g\|_{L^{1}}+\hat{L}\left(\left\|\nu_{\bar{K}}\right\|_{L^{1}\left(J_{m}\right)}+\frac{1}{m}\left\|v_{\bar{K}}\right\|_{L^{1}}\right)\right) \mathcal{S} .
$$

Since we have, according to $\left(2_{\mathrm{iii}}\right), \frac{T+4}{4}\|g\|_{L^{1}}<1$, we can choose $m_{0} \in \mathbb{N}$ such that, for all $m \in \mathbb{N}, m \geq m_{0}$, we have

$$
\frac{T+4}{4}\left(\|g\|_{L^{1}}+\hat{L}\left(\left\|v_{\bar{K}}\right\|_{L^{1}\left(J_{m}\right)}+\frac{1}{m}\left\|v_{\bar{K}}\right\|_{L^{1}}\right)\right)<1 .
$$


Therefore, we get, for sufficiently large $m \in \mathbb{N}$, the contradiction $\mathcal{S}<\mathcal{S}$ which ensures the validity of condition (iii) in Proposition 2.4.

$a d$ (iv) For all $q \in Q$, the set $\mathfrak{T}_{m}(q, 0)$ coincides with the unique solution $x_{m}$ of the linear system

$$
\left.\begin{array}{l}
\ddot{x}(t)=v_{\bar{K}}(t)\left(\chi_{J_{m}}(t)+\frac{1}{m}\right) \hat{\phi}(q(t)), \quad \text { for a.a. } t \in[0, T], \\
x(T)=x(0)=0 .
\end{array}\right\}
$$

According to (15) and (16), for all $t \in[0, T]$,

$$
x_{m}(t)=\int_{0}^{T} G(t, s) \varphi_{m}(s) d s
$$

and

$$
\dot{x}_{m}(t)=\int_{0}^{T} \frac{\partial}{\partial t} G(t, s) \varphi_{m}(s) d s,
$$

where $\varphi_{m}(t):=v_{\bar{K}}(t)\left(\chi_{J_{m}}(t)+\frac{1}{m}\right) \hat{\phi}(q(t))$.

Since

$$
\left\|\varphi_{m}\right\|_{L^{1}} \leq \max _{x \in \overline{B(\partial K, \varepsilon)}}\|\hat{\phi}(x)\| \cdot\left(\left\|v_{\bar{K}}\right\|_{L^{1}\left(J_{m}\right)}+\frac{\left\|v_{\bar{K}}\right\|_{L^{1}}}{m}\right),
$$

we have, for all $t \in[0, T]$,

$$
\left\|x_{m}(t)\right\| \leq \frac{T^{2}}{4} \cdot \max _{x \in \bar{B}(\partial K, \varepsilon)}\|\hat{\phi}(x)\| \cdot\left(\left\|v_{\bar{K}}\right\|_{\left.L^{1} J_{m}\right)}+\frac{\left\|v_{\bar{K}}\right\|_{L^{1}}}{m}\right) .
$$

Let us now consider $r>0$ such that $r B \subset K$. Then it follows from (21) that we are able to find $m_{0} \in \mathbb{N}$ such that, for all $m \in \mathbb{N}, m \geq m_{0}$, and $t \in[0, T],\left\|x_{m}\right\| \leq r$. Therefore, for all $m \in \mathbb{N}, m \geq m_{0}, \mathfrak{T}_{m}(q, 0) \subset$ Int $Q$, for all $q \in Q$, which ensures the validity of condition (iv) in Proposition 2.4.

$a d(\mathrm{v})$ The validity of the transversality condition (v) in Proposition 2.4 can be proven quite analogously as in [16] (see pp.40-43 in [16]) with the following differences:

- due to the Dirichlet boundary conditions, $t_{0}$ belongs to the open interval $(0, T)$,

- since $A(t)=B(t)=0$, we have $p(t)=-v_{\bar{K}}(t)$.

In this way, we can prove that there exists $m_{0} \in \mathbb{N}$ such that every problem $\left(P_{m}\right)$, where $m \geq m_{0}$, satisfies all the assumptions of Proposition 2.4. This implies that every such $\left(P_{m}\right)$ admits a solution, denoted by $x_{m}$, with $x_{m}(t) \in \bar{K}$, for all $t \in[0, T]$. By similar arguments as in [16], but with the expression $Z(4 Z k+1)$ replaced by $\frac{T}{4}$, according to condition $\left(2_{\mathrm{ii}}\right)$, we can obtain the result that there exists a subsequence, denoted as the sequence, and a function $x \in A C^{1}([0, T], E)$ such that $x_{m} \rightarrow x$ and $\dot{x}_{m} \rightarrow \dot{x}$ in $C([0, T], E)$ and also $\ddot{x}_{m} \rightarrow x$ in $L^{1}([0, T], E)$, when $m \rightarrow \infty$. Thus, a classical closure result (see e.g. [27, Lemma 5.1.1]) guarantees that $x$ is a solution of (2) satisfying $x(t) \in \bar{K}$, for all $t \in[0, T]$, and the sketch of proof is so complete.

The case when $F=F_{1}+F_{2}$, with $F_{1}(t, \cdot, \cdot)$ to be completely continuous and $F_{2}(t, \cdot, \cdot)$ to be Lipschitzian, for a.a. $t \in[0, T]$, represents the most classical example of a map which is 
$\gamma$-regular w.r.t. the Hausdorff measure of non-compactness $\gamma$. The following corollary of Theorem 3.1 can be proved quite analogously as in [3, Example 6.1 and Remark 6.1].

Corollary 3.1 Let $E=H$ be a separable Hilbert space and let us consider the Dirichlet b.v.p.:

$$
\left.\begin{array}{l}
\ddot{x}(t) \in F_{1}(t, x(t), \dot{x}(t))+F_{2}(t, x(t), \dot{x}(t)), \quad \text { for a.a. } t \in[0, T], \\
x(0)=x(T)=0,
\end{array}\right\}
$$

where

(i) $F_{1}:[0, T] \times H \times H \multimap H$ is an upper-Carathéodory, globally measurable, multivalued mapping and $F_{1}(t, \cdot, \cdot): H \times H \multimap H$ is completely continuous, for a.a. $t \in[0, T]$, such that

$$
\left\|F_{1}(t, x, y)\right\| \leq v_{1}\left(t, D_{0}\right)
$$

for a.a. $t \in[0, T]$, all $x \in H$ with $\|x\| \leq D_{0}$, where $D_{0}>0$ is an arbitrary constant, $v_{1} \in L^{1}([0, T],[0, \infty))$, and all $y \in H$,

(ii) $F_{2}:[0, T] \times H \times H \multimap H$ is a Carathéodory multivalued mapping such that

$$
\left\|F_{2}(t, 0,0)\right\| \leq v_{2}(t), \quad \text { for a.a. } t \in[0, T]
$$

where $v_{2} \in L^{1}([0, T],[0, \infty))$, and $F_{2}(t, \cdot, \cdot): H \times H \multimap H$ is Lipschitzian, for a.a. $t \in[0, T]$, with the Lipschitz constant

$$
L<\frac{4}{T(T+4)}
$$

Moreover, suppose that

(iii) there exists $R>0$ such that, for all $x \in H$ with $\|x\|=R, t \in(0, T), y \in H$ and $w \in F_{1}(t, x, y)+F_{2}(t, x, y)$, we have

$$
\langle x, w\rangle>0 .
$$

Then the Dirichlet problem (22) admits, according to Theorem 3.1, a solution $x(\cdot)$ such that $\|x(t)\| \leq R$, for all $t \in[0, T]$.

Remark 3.1 For $F_{2}(t, x, y) \equiv 0$, the completely continuous mapping $F_{1}(t, x, y)$ allows us to make a comparison with classical single-valued results recalled in the Introduction. Unfortunately, our $F_{1}$ in (i) (see also $\left(2_{\mathrm{ii}}\right)$ in Theorem 3.1 ) is the only mapping which is (unlike in [3, Example 6.1 and Remark 6.1], where under some additional restrictions quite liberal growth restrictions were permitted) globally bounded w.r.t. $y \in H$. Furthermore, our sign condition in (iii) is also (unlike again in [3, Example 6.1 and Remark 6.1], where under some additional restrictions the Hartman-type condition like $\left(\mathrm{i}_{\mathrm{H}}\right)$ in the Introduction was employed) the most restrictive among their analogies in [6-13]. On the other hand, because of multivalued upper-Carathéodory maps $F_{1}+F_{2}$ in a Hilbert space which are $\gamma$-regular, our result has still, as far as we know, no analogy at all. 


\section{Illustrative examples}

The first illustrative example of the application of Theorem 3.1 concerns the integrodifferential equation

$$
\begin{aligned}
& u_{t t}(t, x)+\varphi\left(t, x, u_{t}(t, x)\right) \\
& =b(t) u(t, x)+\int_{\mathbb{R}} k(x, y) u(t, y) d y+p\left(\int_{\mathbb{R}} \psi(x) u(t, x) d x\right) f(u(t, x)), \\
& \quad t \in[0, T], x \in \mathbb{R},
\end{aligned}
$$

involving discontinuities in a state variable. In this equation, the non-local diffusion term $\int_{\mathbb{R}} k(x, y) u(t, y) d y$ replaces the classical diffusion behavior given by $u_{x x}(t, x)$. In dispersal models such an integral term takes into account the long-distance interactions between individuals (see e.g. [29]). Moreover, when $\varphi$ is linear in $u_{t}$, (23) can be considered as an alternative version of the classical telegraph equation (see e.g. [30] and the references therein), where the classical diffusivity is replaced by the present non-local diffusivity.

Telegraph equations appear in many fields such as modeling of an anomalous diffusion, a wave propagation phenomenon, sub-diffusive systems or modeling of a pulsate blood flow in arteries (see e.g. [31,32]).

For the sake of simplicity, we will discuss here only the case when $\varphi$ is globally bounded w.r.t. $u_{t}$. On the other hand, for non-strictly localized transversality conditions as in [3], for instance, a suitable linear growth estimate w.r.t. $u_{t}$ can be permitted.

Example 4.1 Let us consider the integro-differential equation (23) with $\varphi:[0, T] \times \mathbb{R} \times$ $\mathbb{R} \rightarrow \mathbb{R}, b:[0, T] \rightarrow \mathbb{R}, k: \mathbb{R} \times \mathbb{R} \rightarrow \mathbb{R}, \psi: \mathbb{R} \rightarrow \mathbb{R}$ and $p: \mathbb{R} \rightarrow \mathbb{R}$. We assume that

(a) $\varphi$ is Carathéodory, i.e. $\varphi(\cdot, x, y)$ is measurable, for all $x, y \in \mathbb{R}$, and $\varphi(t, \cdot, \cdot)$ is continuous, for a.a. $t \in[0, T] ; \varphi(t, x, \cdot)$ is $L(t)$-Lipschitzian with $L \in L^{1}([0, T])$; $|\varphi(t, x, y)| \leq \varphi_{1}(t) \varphi_{2}(x)$, for a.a. $t \in[0, T]$ and all $x, y \in \mathbb{R}$, where $\varphi_{1} \in L^{1}([0, T])$ and $\varphi_{2} \in L^{2}(\mathbb{R}) ; \varphi(t, x, 0) \neq 0$, for all a.a. $t \in[0, T]$ and all $x \in \mathbb{R}$,

(b) $b \in L^{1}([0, T])$ and satisfies $b(t) \geq b_{0}>1$, for a.a. $t \in[0, T]$,

(c) $k \in L^{2}(\mathbb{R} \times \mathbb{R})$ with $\|k\|_{L^{2}(\mathbb{R} \times \mathbb{R})}=1$,

(d) $p(r) \geq 0$, for all $r \in \mathbb{R}$; and there can exist $r_{1}<r_{2}<\cdots<r_{k}$ such that $p(\cdot)$ is continuous, for $r \neq r_{i}$, and $p(\cdot)$ has discontinuities at $r_{i}$, for $i=1, \ldots, k$, with $p\left(r_{i}^{\mp}\right):=\lim _{r \rightarrow r_{i}^{\mp}} p(r) \in \mathbb{R}$,

(e) $f$ is $L$-Lipschitzian; $L>0 ; f(0)=0$; and $x f(x)>0$, for all $x \neq 0$,

(f) $\psi \in L^{2}(\mathbb{R})$ with $\|\psi\|_{L^{2}(\mathbb{R})}=1$.

Since the function $p$ can have some discontinuities, a solution of (23) satisfying the Dirichlet conditions

$$
u(0, x)=u(T, x)=0, \quad \text { for all } x \in \mathbb{R},
$$

will be appropriately interpreted in the sense of Filippov. More precisely, let us define $P$ : $\mathbb{R} \multimap \mathbb{R}$ by the formula

$$
P(r):= \begin{cases}p(r) & \text { if } r \neq r_{i}, \\ {\left[\min \left\{p\left(r_{i}\right), p\left(r_{i}^{-}\right), p\left(r_{i}^{+}\right)\right\}, \max \left\{p\left(r_{i}\right), p\left(r_{i}^{-}\right), p\left(r_{i}^{+}\right)\right\}\right]} & \text {if } r=r_{i}, i=1,2, \ldots, k .\end{cases}
$$


A function $u(t, x)$ is said to be a solution of (23), (24) if $u(t, \cdot) \in L^{2}(R)$, for all $t \in[0, T]$, the map $[0, T] \rightarrow L^{2}(R)$ defined by $t \rightarrow u(t, \cdot)$ is $C^{1}$ if it is a solution of the inclusion

$$
\begin{aligned}
& u_{t t}(t, x)+\varphi\left(t, x, u_{t}(t, x)\right) \\
& \quad \in \int_{\mathbb{R}} k(x, y) u(t, y) d y+b(t) u(t, x)+P\left(\int_{\mathbb{R}} \psi(x) u(t, x) d x\right) f(u(t, x))
\end{aligned}
$$

and if it satisfies (24).

If we further assume the existence of $R>0$ such that

$$
R>\frac{\varphi_{1}(t)}{b_{0}-1}\left\|\varphi_{2}\right\|_{L^{2}(\mathbb{R})}, \quad \text { for a.a. } t \in[0, T],
$$

and that

$$
\|L(t)+b(t)\|_{L^{1}([0, T])}+(1+m L) T<\frac{4}{T+4},
$$

where

$$
m:=\max _{r \in[-R, R]} \max \left\{p(r), p\left(r^{-}\right), p\left(r^{+}\right)\right\}
$$

then the problem (23), (24) has a solution, in the sense of Filippov, satisfying $\|u(t, \cdot)\|_{L^{2}(\mathbb{R})} \leq$ $R$, for a.a. $t \in[0, T]$.

In fact, problem (25), (24) can be transformed into the abstract setting

$$
\left\{\begin{array}{l}
\ddot{y}(t) \in F(t, y(t), \dot{y}(t)), \quad t \in[0, T], \\
y(T)=y(0)=0,
\end{array}\right.
$$

where $y(t):=u(t, \cdot) \in L^{2}(\mathbb{R})$, for all $t \in[0, T]$, and $F:[0, T] \times L^{2}(\mathbb{R}) \times L^{2}(\mathbb{R}) \multimap L^{2}(\mathbb{R})$ is defined by

$$
F(t, y, w):=-\hat{\varphi}(t, w)+b(t) y+K(y)+\hat{F}(y)
$$

where $\hat{\varphi}:[0, T] \times L^{2}(\mathbb{R}) \rightarrow L^{2}(\mathbb{R}),(t, y) \mapsto(x \mapsto \varphi(t, x, y(x))), K: L^{2}(\mathbb{R}) \rightarrow L^{2}(\mathbb{R}), w \mapsto$ $\left(x \mapsto \int_{\mathbb{R}} k(x, y) w(y) d y\right), \hat{f}: L^{2}(\mathbb{R}) \rightarrow L^{2}(\mathbb{R}), y \mapsto(x \mapsto f(y(x)))$ and $\hat{F}: L^{2}(\mathbb{R}) \multimap L^{2}(\mathbb{R}), y \multimap$ $\left\{p \hat{f}(y): p \in P\left(\int_{\mathbb{R}} \psi(x) y(x) d x\right)\right\}$.

Let us now examine the properties of $F$. According to (a), $\hat{\varphi}$ is well defined. Given $y \in L^{2}(\mathbb{R})$, let us show that $\hat{\varphi}(\cdot, y)$ is measurable. For this purpose, let $\Psi$ be an arbitrary element in the dual space $\left(L^{2}(\mathbb{R})\right)^{\prime}$ of $L^{2}(\mathbb{R})$. Hence, there exists $\psi \in L^{2}(\mathbb{R})$ such that $\Psi(z)=$ $\int_{\mathbb{R}} \psi(x) z(x) d x$, for all $z \in L^{2}(\mathbb{R})$, and consequently the composition $\Psi \circ \hat{\varphi}(\cdot, y):[0, T] \rightarrow \mathbb{R}$ is such that $t \rightarrow \int_{\mathbb{R}} \psi(x) \varphi(t, x, y(x)) d x$. Since $\varphi$ is Carathéodory, it is globally measurable, and so the mapping $(t, x) \rightarrow \psi(x) \varphi(t, x, y(x))$ is globally measurable as well. This implies that, according to the Fubini Theorem, the mapping $\Psi \circ \hat{\varphi}(\cdot, y)$ is measurable, too. Finally, since $\Psi$ was arbitrary, according to the Pettis Theorem (see Proposition 2.1), $\hat{\varphi}(\cdot, y)$ is measurable.

Furthermore, let us show that $\hat{F}$ is u.s.c. For this purpose, let $y_{0} \in L^{2}(\mathbb{R})$ be fixed. 
(i) If $r_{0}:=\int_{\mathbb{R}} \psi(x) y_{0}(x) d x \neq r_{i}, i=1,2, \ldots, k$, then it is possible to find $\delta>0$ such that $\hat{F}: B\left(y_{0}, \delta\right) \rightarrow L^{2}(\mathbb{R})$ is single-valued, i.e. $\hat{F}(y)=p(r) \hat{f}(y)$,

$r:=\int_{\mathbb{R}} \psi(x) y(x) d x \in\left[r_{0}-\delta, r_{0}+\delta\right]$, for all $y \in B\left(y_{0}, \delta\right)$ and $r_{i} \notin\left[r_{0}-\delta, r_{0}+\delta\right]$, for $i=1,2, \ldots, k$. Since $p$ is continuous in $\left[r_{0}-\delta, r_{0}+\delta\right]$ and $\hat{f}$ is Lipschitzian, $\hat{F}$ is continuous in $B\left(y_{0}, \delta\right)$.

(ii) Let $r_{0}=r_{j}$, for some $j \in\{i=1,2, \ldots, k\}$ and let $U \subset L^{2}(\mathbb{R})$ be open and such that $\hat{F}\left(y_{0}\right) \subset U$. Moreover, let $\sigma>0$ be such that $r:=\int_{\mathbb{R}} \psi(x) y(x) d x \neq r_{i}, i \neq j$, for any $y \in B\left(y_{0}, \sigma\right)$. This implies that $\hat{F}(y)$ is equal either to $p(r) \hat{f}(y)$ or to $P\left(r_{j}\right) \hat{f}(y)$, for all $y \in B\left(y_{0}, \sigma\right)$. If $r<r_{j}$ is such that $\hat{F}(y)=p(r) \hat{f}(y)$, then

$$
\begin{aligned}
\left\|\hat{F}(y)-p\left(r_{j}^{-}\right) \hat{f}\left(y_{0}\right)\right\|_{L^{2}(\mathbb{R})} & =\left\|p(r) \hat{f}(y)-p\left(r_{j}^{-}\right) \hat{f}\left(y_{0}\right)\right\|_{L^{2}(\mathbb{R})} \\
& \leq\left|p(r)-p\left(r_{j}^{-}\right)\right| \cdot\|\hat{f}(y)\|+p\left(r_{j}^{-}\right) \cdot\left\|\hat{f}(y)-\hat{f}\left(y_{0}\right)\right\|,
\end{aligned}
$$

which implies that it is possible to find $\sigma_{1}>0$ such that $F(y) \subset U$, for all $y \in B\left(y_{0}, \sigma_{1}\right)$. Similarly, we would obtain the same when assuming $r>r_{j}$. If $\hat{F}(y)=P\left(r_{j}\right) \hat{f}(y)$ then, for every $p \in P\left(r_{j}\right)$,

$$
\left\|p \hat{f}(y)-p \hat{f}\left(y_{0}\right)\right\|_{L^{2}(\mathbb{R})}=|p| \cdot\left\|\hat{f}(y)-\hat{f}\left(y_{0}\right)\right\| \leq m\left\|\hat{f}(y)-\hat{f}\left(y_{0}\right)\right\|,
$$

which implies that also in this case it is possible to find $\sigma_{2}>0$ such that $F(y) \subset U$, for all $y \in B\left(y_{0}, \sigma_{2}\right)$.

Moreover, according to (a) and (c), $\hat{\varphi}$ is a Carathéodory mapping such that $\hat{\varphi}(t, \cdot)$ is $L(t)$ Lipschitzian, for all $t \in[0, T]$, and $K$ is well defined and 1-Lipschitzian. It can also be shown that, according to (d) and (e), $\hat{F}$ has compact and convex values. Therefore, the mapping $F$ is globally measurable, and so has the Scorza-Dragoni property (cf. Proposition 2.2).

Let us now verify particular assumptions of Theorem 3.1.

Let $\Omega_{1} \subset\left\{y \in L^{2}(\mathbb{R}) \mid\|y\|_{L^{2}(\mathbb{R})} \leq R\right\}$. Then, according to (f),

$$
\int_{\mathbb{R}} \psi(x) y(x) d x \in[-R, R]
$$

for all $y \in \Omega_{1}$. Hence,

$$
\begin{aligned}
\hat{F}\left(\Omega_{1}\right) & =\left\{p \hat{f}(y): p \in P\left(\int_{\mathbb{R}} \psi(x) y(x) d x\right), y \in \Omega_{1}\right\} \subset\left\{p \hat{f}\left(\Omega_{1}\right): p \in[0, m]\right\} \\
& =\left\{m \cdot \alpha \cdot \hat{f}\left(\Omega_{1}\right): \alpha \in[0,1]\right\},
\end{aligned}
$$

where $m$ is defined by (28).

Thus,

$$
\gamma\left(\hat{F}\left(\Omega_{1}\right)\right) \leq m \gamma\left(\left\{\alpha \cdot \hat{f}\left(\Omega_{1}\right): \alpha \in[0,1]\right\}\right) \leq m \cdot L \cdot \gamma\left(\Omega_{1}\right),
$$

according to the Lipschitzianity of $\hat{f}$ and property (6). For a.a. $t \in[0, T]$ and all $\Omega_{2} \subset L^{2}(\mathbb{R})$, we have

$$
\begin{aligned}
\gamma\left(F\left(t, \Omega_{1} \times \Omega_{2}\right)\right) & \leq \gamma\left(\hat{\varphi}\left(t, \Omega_{2}\right)\right)+\gamma\left(b(t) \Omega_{1}\right)+\gamma\left(K\left(\Omega_{1}\right)\right)+\gamma\left(\hat{F}\left(\Omega_{1}\right)\right) \\
& \leq L(t) \gamma\left(\Omega_{2}\right)+b(t) \gamma\left(\Omega_{1}\right)+\gamma\left(\Omega_{1}\right)+m \cdot L \cdot \gamma\left(\Omega_{1}\right),
\end{aligned}
$$


and so condition $\left(2_{\mathrm{i}}\right)$ is satisfied with $g(t)=L(t)+b(t)+1+m \cdot L$. The obtained form of $g(t)$ together with assumption (27) directly guarantee the condition $\left(2_{\mathrm{iii}}\right)$. It can also be easily shown that properties of $F$ ensure the validity of condition $\left(2_{\mathrm{ii}}\right)$.

In order to verify conditions imposed on a bounding function, let us define $V: L^{2}(\mathbb{R}) \rightarrow$ $\mathbb{R}, \alpha \rightarrow \frac{1}{2}\left(\|\alpha\|_{L^{2}(\mathbb{R})}^{2}-R^{2}\right)$. The function $V \in C^{2}\left(L^{2}(\mathbb{R}), \mathbb{R}\right)$ with $\dot{V}_{x}: h \rightarrow\langle x, h\rangle$ obviously satisfies (10), so it is only necessary to check condition (11). Thus, let $\alpha \in L^{2}(\mathbb{R}),\|\alpha\|_{L^{2}(\mathbb{R})}=$ $R, t \in(0, T), v \in L^{2}(\mathbb{R})$ and $z \in F(t, \alpha, v)$. Then there exists $p^{*} \in P\left(\int_{\mathbb{R}} \psi(x) \alpha(x) d x\right)$ such that

$$
z=-\hat{\varphi}(t, v)+b(t) \alpha+K(\alpha)+p^{*} \hat{f}(\alpha) .
$$

Moreover, since $p^{*} \geq 0$ and $\int_{\mathbb{R}} \alpha(x) f(\alpha(x)) d x \geq 0$, we see that

$$
p^{*} \int_{\mathbb{R}} \alpha(x) f(\alpha(x)) d x \geq 0,
$$

and since

$$
\left|\int_{\mathbb{R}} \alpha(x) \int_{\mathbb{R}} k(x, y) \alpha(y) d y d x\right| \leq R \int_{\mathbb{R}}|\alpha(x)| \cdot\|k(x, y)\|_{L^{2}(\mathbb{R})} d x \leq R^{2},
$$

we see that

$$
\int_{\mathbb{R}} \alpha(x) \int_{\mathbb{R}} k(x, y) \alpha(y) d y d x \geq-R^{2} .
$$

The properties (a)-(f) together with the well-known Hölder inequality then yield

$$
\begin{aligned}
\left\langle\dot{V}_{\alpha}, z\right\rangle= & \langle\alpha, z\rangle \\
= & -\int_{\mathbb{R}} \alpha(x) \varphi(t, x, v(x)) d x+b(t) \int_{\mathbb{R}} \alpha^{2}(x) d x \\
& +\int_{\mathbb{R}} \alpha(x) \int_{\mathbb{R}} k(x, y) \alpha(y) d y d x+p^{*} \int_{\mathbb{R}} \alpha(x) f(\alpha(x)) d x \\
\geq & -R \varphi_{1}(t)\left\|\varphi_{2}\right\|_{L^{2}(\mathbb{R})}+b_{0} R^{2}-R^{2}>0,
\end{aligned}
$$

in view of condition (26), (30), and (31).

Hence, the Dirichlet problem (29) admits, according to Theorem 3.1, a solution $y$ satisfying $\|y(t)\|_{L^{2}(\mathbb{R})} \leq R$, for a.a. $t \in(0, T)$. If $u(t, x):=y(t)(x)$, then $u$ is a solution of (24), (25) which is the Filippov solution of the original problem (23), (24).

Finally, we can sum up the above result in the form of the following theorem.

Theorem 4.1 Let the assumptions (a)-(f) be satisfied. If still conditions (26), (27) hold, then the problem (23), (24) admits a non-trivial solution $u$ in the sense of Fillippov such that $\|u(t, \cdot)\|_{L^{2}(\mathbb{R})} \leq R$.

Remark 4.1 In [13, Example 5.2], the following formally simpler integro-differential equation in $\mathbb{R}$ :

$$
u_{t t}(t, x)=\int_{0}^{1} \tilde{k}(x, y) u(t, y) d y, \quad t \in(0,1), x \in[0,1],
$$


with non-homogeneous Dirichlet conditions

$$
u(0, x)=u_{0}(x), \quad u(1, x)=u_{1}(x), \quad x \in[0,1], u_{0}, u_{1} \in L^{2}([0,1])
$$

was solved provided $\tilde{k}:[0,1] \times[0,1] \rightarrow(0, \infty)$ is a positive kernel of the Hilbert-Schmidttype and the norms $\left\|u_{0}\right\|_{L^{2}([0,1])}$ and $\left\|u_{1}\right\|_{L^{2}([0,1])}$ are finite.

After the homogenization of boundary conditions, the Dirichlet problem takes the form

$$
\begin{aligned}
& u_{t t}(t, x)=\tilde{\varphi}(t, x)+\int_{0}^{1} \tilde{k}(x, y) u(t, y) d y, \quad t \in(0,1), x \in[0,1], \\
& u(0, x)=u(1, x)=0
\end{aligned}
$$

where $\tilde{\varphi}(t, x):=\int_{0}^{1} \tilde{k}(x, y)\left\{\left[u_{1}(y)-u_{0}(y)\right] t+u_{0}(y)\right\} d y$.

Thus, it can be naturally extended onto the infinite strip $[0,1] \times \mathbb{R}$, into the form (23), (24), where

$$
\begin{aligned}
& \varphi(t, x, y) \equiv \varphi(t, x):= \begin{cases}-\tilde{\varphi}(t, x) & \text { if }(t, x) \in(0,1) \times[0,1], \\
0 & \text { otherwise, }\end{cases} \\
& k(t, z):= \begin{cases}\tilde{k}(x, z) & \text { if }(x, z) \in[0,1] \times[0,1], \\
0 & \text { otherwise, }\end{cases}
\end{aligned}
$$

and $b(t) \equiv 0, p(r) \equiv 0$ or $f(s) \equiv 0$.

The result in [13, Example 5.2] cannot be, however, deduced from Theorem 3.1, because condition (b) in Example 4.1 cannot be satisfied in this way.

On the other hand, the linear term with coefficient $b$ could not be implemented in their equation, because it is not completely continuous in (36) below, as required in [13].

In view of the arguments in Remark 4.1, we can conclude by the second illustrative example.

Example 4.2 Consider the following non-homogeneous Dirichlet problem in $\mathbb{R}$ :

$$
\left.\begin{array}{l}
u_{t t}(t, x)=b(t) u(t, x)+\int_{0}^{1} \tilde{k}(x, y) u(t, y) d y, \quad t \in(0,1), x \in[0,1] \\
u(0, x)=u_{0}(x), \quad u(1, x)=u_{1}(x), \quad x \in[0,1], u_{0}, u_{1} \in L^{2}([0,1]),
\end{array}\right\}
$$

where $\tilde{k}:[0,1] \times[0,1] \rightarrow(0, \infty)$ is a positive kernel of the Hilbert-Schmidt-type such that

$$
k_{0}:=\|\tilde{k}\|_{L^{2}([0,1] \times[0,1])}<\infty
$$

and $b \in L^{1}((0,1))$ is such that $b(t) \geq b_{0}>0$, for a.a. $t \in(0,1)$.

Furthermore, let there exist a constant $L<\frac{4}{5}$ such that

$$
\underset{t \in(0,1)}{\operatorname{ess} \sup } b(t) \leq L
$$

The properties of $u_{0}$ and $u_{1}$ guarantee that there exists $B \geq 0$ such that

$$
\left\|u_{0}-u_{1}\right\|_{L^{2}([0,1])}+\left\|u_{0}\right\|_{L^{2}([0,1])} \leq B
$$


We will show that, under (33) and (34), problem (32) is solvable, in the abstract setting, by means of Corollary 3.1 .

Problem (32) can be homogenized as follows:

$$
\left.\begin{array}{l}
\hat{u}_{t t}(t, x)=\varphi(t, x)+b(t) \hat{u}(t, x)+\int_{0}^{1} \tilde{k}(x, y) \hat{u}(t, y) d y, \quad t \in(0,1), x \in[0,1], \\
\hat{u}(0, x)=\hat{u}(1, x)=0,
\end{array}\right\}
$$

where

$$
\varphi(t, x):=b(t)\left\{\left[u_{1}(x)-u_{0}(x)\right] t+u_{0}(x)\right\}+w(t, x), \quad t \in(0,1), x \in[0,1],
$$

with $w(t, x):=\int_{0}^{1} \tilde{k}(x, y)\left\{\left[u_{1}(y)-u_{0}(y)\right] t+u_{0}(y)\right\} d y$.

Since the Hilbert-Schmidt operator

$$
\int_{0}^{1} \bar{k}(y)(\cdot) d y: L^{2}([0,1]) \rightarrow L^{2}([0,1])
$$

where $\bar{k}(y)(\cdot):=\tilde{k}(x, y)$ is well known to be completely continuous (cf. [13, Example 5.2]) and $b(t)(\cdot): L^{2}([0,1]) \rightarrow L^{2}([0,1])$ is, according to (33), $L$-Lipschitzian with $L<\frac{4}{5}$, conditions (i), (ii) in Corollary 3.1 can be easily satisfied, for $\bar{u}(t):=u(t, x), \bar{u} \in L^{2}([0,1])$,

$$
F_{1}(t, \bar{u}, \bar{v}) \equiv F_{1}(t, \bar{u}):=\bar{\varphi}(t)+f(\bar{u}),
$$

where $\bar{\varphi}(t):=b(t)\left\{\left[\bar{u}_{1}-\bar{u}_{0}\right] t+\bar{u}_{0}\right\}+\bar{w}(t), \bar{w}(t):=w(t, x)$,

$$
f(\bar{u}):=\int_{0}^{1} \bar{k}(y) \bar{u}(y) d y
$$

and

$$
F_{2}(t, \bar{u}, \bar{v}) \equiv F_{2}(t, \bar{u}):=b(t) \bar{u} .
$$

In this setting, problem (35) takes the abstract form as (22), namely

$$
\left.\begin{array}{l}
\ddot{\bar{u}}(t)=f(\bar{u}(t))+b(t) \bar{u}(t)+\bar{\varphi}(t), \quad \text { for a.a. } t \in(0,1), \\
\bar{u}(0)=\bar{u}(1)=0 .
\end{array}\right\}
$$

Since $\langle f(\bar{u}), \bar{u}\rangle \geq 0$ holds, for all $\bar{u} \in L^{2}([0,1])$ (see [13, Example 5.2]) one can check that the strict inequality in (iii) in Corollary 3.1 can be easily satisfied, for (32), whenever

$$
R>\frac{B\left(L+k_{0}\right)}{b_{0}} .
$$

Hence, applying Corollary 3.1, problem (36) admits a solution, say $\hat{u}(\cdot)$, such that

$$
\|\hat{u}\|_{L^{2}([0,1])} \leq R,
$$


where $R$ satisfies (37), and subsequently the same is true for (32), i.e.

$$
\max _{t \in[0,1]}\|\hat{u}(t, \cdot)\|_{L^{2}([0,1])} \leq R,
$$

as claimed.

After all, we can sum up the sufficient conditions for the existence of a solution $\hat{u}$ of (32) satisfying (38) as follows:

- $\tilde{k}$ is a positive kernel of the Hilbert-Schmidt operator with the finite norm

$$
k_{0}:=\|\tilde{k}\|_{L^{2}([0,1] \times[0,1])}<\infty,
$$

- there exists $b_{0}>0, L<\frac{4}{5}: b_{0} \leq b(t) \leq L$, for a.a. $t \in(0,1)$,

- condition (37) holds.

\section{Competing interests}

The authors declare that they have no competing interests.

\section{Authors' contributions}

All authors contributed equally in this article. They read and approved the final manuscript.

\section{Author details}

'Department of Mathematical Analysis and Applications of Mathematics, Faculty of Science, Palacký University, 17. listopadu 12, Olomouc, 771 46, Czech Republic. ${ }^{2}$ Department of Sciences and Methods for Engineering, University of Modena and Reggio Emilia, Via G. Amendola, 2 - pad. Morselli, Reggio Emilia, 42122, Italy.

\section{Acknowledgements}

The first and third authors were supported by grant 'Singularities and impulses in boundary value problems for non-linear ordinary differential equations'. The second author was supported by the national research project PRIN 'Ordinary Differential Equations and Applications'

\section{Endnotes}

a The m.n.c. $\bmod _{C}(\Omega)$ is a monotone, non-singular and algebraically subadditive on $C([0, T], E)(c f$. e.g. $[27])$ and it is equal to zero if and only if all the elements $x \in \Omega$ are equi-continuous.

b Since a $C^{2}$-function $V$ has only a locally Lipschitzian Fréchet derivative $\dot{V}$ (cf. e.g. [21]), we had to assume explicitly the global Lipschitzianity of $\dot{V}$ in a non-compact set $\overline{B(\partial K, \varepsilon)}$.

Received: 16 October 2013 Accepted: 9 January 2014 Published: 27 Jan 2014

\section{References}

1. Hartman, P: Ordinary Differential Equations. Willey, New York (1964)

2. Scorza-Dragoni, G: Un teorema sulle funzioni continue rispetto ad una e misurabili rispetto ad un'altra variabile. Rend. Semin. Mat. Univ. Padova 17, 102-106 (1948)

3. Andres, J, Malaguti, L, Pavlačková, M: Dirichlet problem in Banach spaces: the bound sets approach. Bound. Value Probl. 2013, 25 (2013)

4. Scorza-Dragoni, G: Sui sistemi di equazioni integrali non lineari. Rend. Semin. Mat. Univ. Padova 7, 1-35 (1936)

5. Scorza-Dragoni, G: Sul problema dei valori ai limiti per i systemi di equazioni differenziali del secondo ordine. Boll. Unione Mat. Ital. 14, 225-230 (1935)

6. Amster, P, Haddad, J: A Hartman-Nagumo type conditions for a class of contractible domains. Topol. Methods Nonlinear Anal. 41(2), 287-304 (2013)

7. Gaines, RE, Mawhin, J: Coincidence Degree, and Nonlinear Differential Equations. LNM, vol. 568. Springer, Berlin (1977)

8. Granas, A, Guenther, RB, Lee, JW: Some existence principles in the Carathéodory theory of nonlinear differentia system. J. Math. Pures Appl. 70, 153-196 (1991)

9. Hartman, P: On boundary value problems for systems of ordinary differential equations. Trans. Am. Math. Soc. 96, 493-509 (1960)

10. Lasota, A, Yorke, JA: Existence of solutions of two-point boundary value problems for nonlinear systems. J. Differ. Equ. 11(3), 509-518 (1972)

11. Mawhin, J: Boundary value problems for nonlinear second order vector differential equations. J. Differ. Equ. 16 257-269 (1974)

12. Mawhin, J: Two point boundary value problems for nonlinear second order differential equations in Hilbert spaces Tohoku Math. J. 32, 225-233 (1980) 
13. Schmitt, K, Thompson, RC: Boundary value problems for infinite systems of second-order differential equations. J. Differ. Equ. 18(2), 277-295 (1975)

14. Mawhin, J: The Bernstein-Nagumo problem and two-point boundary value problems for ordinary differential equations. In: Farkas, M (ed.) Qualitative Theory of Differential Equations, Budapest, pp. 709-740 (1981)

15. Pavlačková, M: A Scorza-Dragoni approach to Dirichlet problem with an upper-Carathéodory right-hand side. Topol. Methods Nonlinear Anal. (to appear)

16. Andres, J, Malaguti, L, Pavlačková, M: A Scorza-Dragoni approach to second-order boundary value problems in abstract spaces. Appl. Math. Inform. Sci. 6(2), 177-192 (2012)

17. Andres, J, Malaguti, L, Taddei, V: On boundary value problems in Banach spaces. Dyn. Syst. Appl. 18, $275-302$ (2009)

18. Cecchini, S, Malaguti, L, Taddei, V: Strictly localized bounding functions and Floquet boundary value problems. Electron. J. Qual. Theory Differ. Equ. 2011, 47 (2011)

19. Andres, J, Malaguti, L, Pavlačková, M: On second-order boundary value problems in Banach spaces: a bound sets approach. Topol. Methods Nonlinear Anal. 37(2), 303-341 (2011)

20. Andres, J, Väth, M: Coincidence index for noncompact mappings on nonconvex sets. Nonlinear Funct. Anal. Appl. 7(4), 619-658 (2002)

21. Papageorgiou, NS, Kyritsi-Yiallourou, ST: Handbook of Applied Analysis. Springer, Berlin (2009)

22. Rzezuchowski, T: Scorza-Dragoni type theorem for upper semicontinuous multivalued functions. Bull. Acad. Pol. Sci., Sér. Sci. Math. 28(1-2), 61-66 (1980)

23. Andres, J, Górniewicz, L: Topological Fixed Point Principles for Boundary Value Problems. Topological Fixed Point Theory and Its Applications, vol. 1. Kluwer Academic, Dordrecht (2003)

24. Pettis, BJ: On the integration in vector spaces. Trans. Am. Math. Soc. 44(2), 277-304 (1938)

25. Bader, R, Kryszewski, W: On the solution set of differential inclusions and the periodic problem in Banach spaces. Nonlinear Anal. 54(4), 707-754 (2003)

26. Hu, S, Papageorgiou, NS: Handbook of Multivalued Analysis. Volume l: Theory. Kluwer Academic, Dordrecht (1997)

27. Kamenskii, MI, Obukhovskii, WV, Zecca, P: Condensing Multivalued Maps and Semilinear Differential Inclusions in Banach Spaces. de Gruyter, Berlin (2001)

28. Deimling, K: Multivalued Differential Equations. de Gruyter, Berlin (1992)

29. Jin, Y, Zhao, X: Spatial dynamics of a periodic population model with dispersal. Nonlinearity 22, 1167-1189 (2009)

30. Alonso, JM, Mawhin, J, Ortega, R: Bounded solutions of second order semilinear evolution equations and applications to the telegraph equation. J. Math. Pures Appl. 78, 49-63 (1999)

31. Jeffrey, A: Advanced Engineering Mathematics. Harcourt Academic Press, Burlington (2002)

32. Pozar, DM: Microwave Engineering. Adison-Wesley, New York (1990)

10.1186/1687-2770-2014-23

Cite this article as: Andres et al.: Scorza-Dragoni approach to Dirichlet problem in Banach spaces. Boundary Value Problems 2014, 2014:23

\section{Submit your manuscript to a SpringerOpen ${ }^{\ominus}$ journal and benefit from:}

- Convenient online submission

- Rigorous peer review

- Immediate publication on acceptance

- Open access: articles freely available online

- High visibility within the field

- Retaining the copyright to your article 